\title{
On dynamic interactions between body motion and fluid motion
}

\author{
Frank T. Smith, Samire Balta, Kevin Liu, and Edward R. Johnson \\ University College London, London WC1E 6BT, UK, \\ samire.balta.12@ucl.ac.uk
}

\begin{abstract}
This contribution on dynamic fluid-body interactions concentrates on applying mathematical/analytical ideas to complement direct numerical studies. The typical body may be of given shape or flexible depending on the context. In the background there are numerous realworld motivations in industry, biomedical and environmental applications, many of which involve high flow rates. A review of ideas developed over the last decade for cases of high flow rates first addresses inviscid approaches to one or more bodies free to move within a channel flow, a skimming sharp-edged body on a free surface, the sinking of a body in water and the rocking or rolling of a body on a solid surface, before moving on to more recent viscous-inviscid approaches for channel flows and boundary layers. The beginnings of certain current research projects are also outlined. These concern models of liftoff of a body from a solid surface, the impact of a smooth body during skimming and viscous-inviscid effects in the presence of more than one freely moving body. Linear and nonlinear mathematical properties as appropriate are described.
\end{abstract}

Keywords: fluid-solid interactions, channel flow, skimming, lift-off

\section{Introduction}

Dynamic fluid-body interactions where the body and fluid motions generally affect each other about equally [1]-[11] are the basis for this chapter, with some review and some new developments. The motivation comes from applications as well as scientific interest. There have been a fair number of computational, experimental or observational studies in the area [12]-[22] but comparatively few analytical studies; the latter tend to lead to complementary understanding of parametric and physical effects. Our aim indeed is to combine modelling, analysis, reduced computation and experimental links where possible. The present contribution addresses several different scenarios, for example with viscous or inviscid fluid, with internal or external flow, with a free surface or without.

Applications and motivation. The study of interactions between moving solid or flexible bodies (including particles, grains) and the surrounding fluid has many environmental, industrial and biomedical applications. Numerous applications arise across nature such as with falling leaves and moving seeds and coffee 
grains, not to forget the motion of frozen ice particles and hailstones as well as sedimentation and fluidisation phenomena. Applications also arise in sporting contexts such as running and cycling groups and to some extent in swimming competitions and in surfboarding. The behaviours of various swarms similarly have an interactive fluid-dynamical element to them. Three industrial applications are concerned with the falling of lumps of ice into an engine intake in an aerodynamic safety context, the travel of wind-blown particles of ice along a wing surface again in the aerodynamic safety context and the falling of rice grains down a chute in a food-sorting context. In addition various disintegration, deposition, liftoff, surface cleansing, oil-well and sequestration modelling applications exist for interactions between solid bodies and fluids. There are also many biomedical applications in principle, for example on travel of solids within vessels of major networks in the human body. Specific applications are to transport of blood clots, embolisation procedures in stroke treatment (transportation of glue), drug-delivery to tumours via a capillary network, the passage of cells through vessels of lateral dimension comparable with the effective cell diameter, and deposition of tiny particles in branching systems [23]-[30]. One fundamental question is how far and where small objects will travel when transported, which is a global network issue as well as depending on the shapes of the objects and the local vessel shapes.

The solid-fluid interactions of interest are with or without side walls being present. One specific example is in food-sorting where grains travel as a monolayer down an inclined chute (which has a free-surface top), fall from the bottom of the chute and then pass an optical system that can detect defective grains. A powerful jet of air is fired to eject a defective grain. Since the grains falling off the chute typically are not uniformly distributed but clustered and inhomogeneous, the air jet removes other grains surrounding the target grain which may not themselves be defective. An industrial goal is to increase uniformity of product feed to reduce the ejection difficulty whilst maintaining a high throughput of grains. The ideal situation is for an evenly spaced and uniformly ordered array of grains to fall down and off the chute such that each grain is aligned with an ejector. This points to a study of air effects on arrays of grains. Numerical estimates on food-sorting in [31], [32] indicate that an inviscid approximation is reasonable as a first-go model.

Background studies. The most relevant studies are in [1]-[10] on interactions for channels, boundary layers, free surfaces, liftoff. We should mention in addition suspension flow studies concerned with relatively sparse grain flows where the interstitial fluid is important for the grain dynamics, such as in aeolian or fluvial transport [1], [33], [34]. These studies address issues such as entrainment which are potentially relevant to some fluid-body interactions of present concern. Other investigations consider oscillations in sedimentation of spheres and fibres, bubbles in fluidized beds, migration of particles, bubble formations and clusters (see [1]). Nonlinear multi-body interactions addressed [35]-[37] for in-series wakes and internal branching motions are also relevant although they assume 
fixed bodies in steady flow.

The present article. We will begin below with the original food-sorting context in mind before branching out to skimming, liftoff, viscous influences and related configurations. The rapid monolayers in the sorting applications are atypical for a granular flow, and enduring contacts are not as significant here; frequent binary impacts or clashes are more typical. The issues involved lie between or outside the arenas of existing granular flows and suspension flows. The current focus is on substantial interactions in which the fluid flow is at relatively high rates, tending to produce a flow multi-structure. The bodies (grains) respond nonlinearly by means of their own induced motion which in turn affects the fluid flow nonlinearly. In terms of grains falling down and off a chute for example we concentrate first on the fluid-body interactions occurring at the lower end of the chute where the fluid response is effectively inviscid due to the increased velocity of the bodies. The upper part of the chute where viscous or viscous-inviscid behaviour is more appropriate is considered in [31] (see also section 2 below), while a continuum model for the bulk properties of the grain motion without air effects is in [32]. Similar considerations apply to other contexts.

Section 2 describes the model situations of interest, assuming laminar unsteady incompressible fluid flow. These have fluid-body interactions in two spatial dimensions for: one or more bodies inside a channel; skimming of a body along a free surface; rocking of a body on a solid surface; viscous-inviscid effects within a channel or boundary-layer flow. In each case the interaction model and solution structure lead to a nonlinear system of difference, longitudinaldifferential and /or integral equations for the motion of one or a finite number of bodies in surrounding fluid or on a free surface. Section 3 then examines more recent developments in the skimming scenario. The phenomenon of take-off of a body from a solid surface is examined in detail in section 4 . The influences of many bodies and viscous effects then form the motivation for the work in section 5 , with the viscous effects leading to other scales coming into play. Further comments including future research possibilities are made in section 6 .

\section{The models}

We consider the four main model areas in sections 2.1-2.4 below, with the first section giving the typical form of non-dimensionalisation used throughout.

\subsection{Bodies in a channel flow}

The configuration of concern here models grains falling through fluid down a vertical chute as in the introduction but is drawn rotated in figure 1 giving thin bodies which travel almost horizontally. The entire motion takes place in a planar Cartesian frame $\left(x^{*}, y^{*}\right)$ as shown, with an asterisk signifying a dimensional quantity. The leading edges of the bodies are aligned with each other and their trailing edges with each other. The bodies form an unknown row-like pattern 
and they are finite in number. The representative axial extent of the bodies from leading to trailing edge is $L_{1}^{*}$ and the lateral distance in $y^{*}$ is $L_{2}^{*}$. The typical axial flow speed $U^{*}$ is a prescribed constant. The velocity components are $u^{*}, v^{*}$ in $x^{*}, y^{*}$ respectively, the pressure is $p^{*}, t^{*}$ denotes the time and $\rho^{*}$ is the fluid density.

Non-dimensional scaled quantities are used in the frame moving with the bodies at speed $U^{*}$, such that

$$
\begin{aligned}
& {\left[\left(x^{*}-x_{0}^{*}\right) / L_{1}^{*}, y^{*} / L_{1}^{*}, t^{*} U^{*} / L_{1}^{*}\right]=[x, \beta \hat{y}, t],} \\
& {\left[u^{*} / U^{*}, v^{*} / U^{*}, p^{*} /\left(\rho^{*} U^{* 2}\right)\right]=\left[u_{n}, \beta \hat{v}_{n}, p_{n}\right],}
\end{aligned}
$$

where $\beta \equiv L_{2}^{*} / L_{1}^{*}$. The location $x_{0}^{*}$ is a constant, as is confirmed later by the equations of body motion. The governing equations in full for the fluid are the continuity and Navier-Stokes equations and for the bodies are those of rigid body motion. The fluid and the bodies interact by virtue of the unknown movements of the individual bodies when subjected to fluid dynamic forces and the equally unknown flow of the fluid affected by the moving boundaries. When the typical length ratio $\beta$ is small the fluid flow equations in each gap become those of thin inviscid layers to leading order,

$$
\begin{gathered}
\partial u_{n} / \partial x+\partial \hat{v}_{n} / \partial \hat{y}=0 \\
\partial u_{n} / \partial t+u_{n} \partial u_{n} / \partial x+\hat{v}_{n} \partial u_{n} / \partial \hat{y}=-\partial p_{n} / \partial x, \\
0=-\partial p_{n} / \partial \hat{y}
\end{gathered}
$$

in the majority of the flow field. Here $u_{n}, \hat{v}_{n}, p_{n}, \hat{y}$ are of order unity, and $n$ runs from 1 to $N$ in the successive $N$ gaps for $(N-1)$ bodies in a row between the side walls $\hat{y}=0$ and $\hat{y}=1$. The integer $N \geq 2$. The equations above come from the balances of continuity, longitudinal momentum and lateral momentum respectively. These hold provided not only that $\beta$ is small but $\beta^{2} R e$ is large, where $R e$ is the characteristic Reynolds number $U^{*} L_{1}^{*} / \nu^{*}$ and $\nu^{*}$ is the kinematic viscosity of the fluid. The negligible inertial impact in (2c) implies that $p_{n}$ is an unknown function of $x, t$ only. The oncoming fluid motion effectively has $u_{n}=1, \hat{v}_{n}=0, p_{n}=0$ due to the frame of reference. Effects of gravity, viscosity and wall-contact are neglected. The boundary conditions are the kinematic ones

$$
\hat{v}_{n}=\partial f_{n}^{ \pm} / \partial t+u_{n} \partial f_{n}^{ \pm} / \partial x \quad \text { on } \quad \hat{y}=f_{n}{ }^{ \pm}(x, t),
$$

on the surface of each body given by the unknown position $f_{n}{ }^{ \pm}(x, t), n=1$ to $(N-1)$, with superscripts $(+,-)$ referring to the upper and lower surface in turn as in figure 1 , and

$$
\hat{v}_{1}=0 \text { on } \hat{y}=0, \quad \hat{v}_{N}=0 \text { on } \hat{y}=1,
$$

for tangential flow at the straight solid side walls. The bodies are closed in the sense that $f_{n}^{+}(0, t)=f_{n}^{-}(0, t), f_{n}^{+}(1, t)=f_{n}^{-}(1, t)$. At the trailing edges 


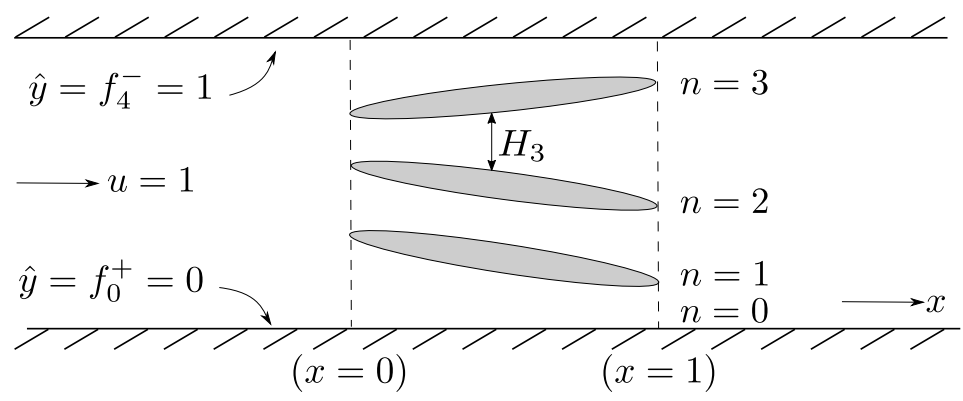

(a)

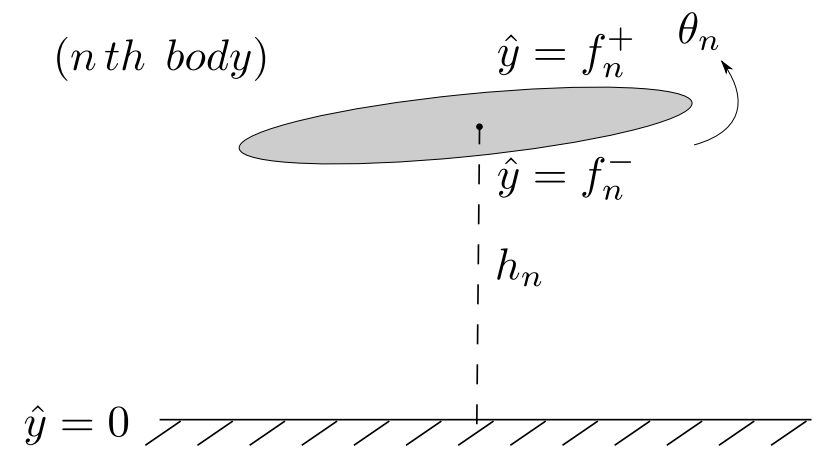

(b)

Fig. 1. (a) Sketch of dynamic fluid-body interaction in a channel, with uniform oncoming flow, in nondimensional terms. There are $N$ gaps and $N-1$ bodies; in this case $N=4$. Representative gap width $H \equiv H_{3}(x, t)=\left(f_{3}^{-}-f_{2}^{+}\right)(x, t)$ is shown. (b) Nomenclature for the typical $n$th body. Here $h_{n}(t)$ is the nondimensional height of the centre of mass and $\theta_{n}(t)$ is the orientation angle. 
where $x$ is unity Kutta conditions apply as the individual gap flows enter into the common wake requiring the pressures across all the gaps to be equal there,

$$
p_{1}=p_{2}=\cdots=p_{N} \quad \text { at } \quad x=1 .
$$

By contrast the velocities $u_{n}$ at the trailing edges are unequal generally, admitting vortex sheets into the common wake.

A significant feature associated with upstream influence is that streamwise jumps (Euler jumps, as in [38]) in pressure must occur at the leading-edge station. The reason stems from the hyperbolic nature of the gap flows in (2a-2c) which indicates zero upstream influence in general and so a possible contradiction with the equi-pressure requirement (2f) at each trailing edge as the different gap flows usually produce different pressures at the trailing edges if they begin with identical leading-edge pressures. The resolution is provided by a flow-solution discontinuity which can occur in a self-consistent manner only in the vicinity of the leading edges, where all the upstream influence is focused in a sense. It follows that in general the incident pressure of zero just ahead of the $n$th leading edge is different from the two distinct values $p_{n}(0+, t), p_{n+1}(0+, t)$ (say $\pi_{n}, \pi_{n+1}$ respectively) holding on either side of the $n$th body just downstream. Instead the scaled Bernoulli quantity $p+1 / 2 u^{2}$ and the scaled vorticity are conserved across the leading-edge station and give rise to the conditions that $p+1 / 2 u^{2}=1 / 2$ and $\partial u / \partial y$ is zero at the onset of each gap flow, in view of the incident uniform stream and pressures. The streamwise length scale to smooth out the jumps is of order $\beta$ in $x$ and the sizes of the velocity and pressure are $u \sim v \sim 1, p \sim 1$. Hence the leading-edge region is controlled by quasi-steady Euler dynamics spanning the channel from wall to wall, with the thin bodies appearing as flat plates aligned with the incident stream, and that scenario leads to conservation of the Bernoulli quantities and (zero) vorticity along each streamline and indeed to Laplaces equation for the scaled stream function. The flow enters and leaves the present Euler region uni-directionally but with an overall displacement of its streamlines accompanied by pressure changes, inside each gap, consistent with the upstream-to-downstream jumps described above, and with smooth attached flow in between such that the stream function is an unknown constant on each of the quasi-semi-infinite bodies. Relatively thin viscous boundary layers are generated on every leading-edge surface and these are supposed to remain attached, before forming the beginnings of a relatively small Blasius-like effect on each body over the longer scale of (2a-2f). Substantial separations at the leading edge and elsewhere are discounted.

Thus the flow in (2a-2f) remains irrotational virtually everywhere and the scaled vorticity is identically zero. Hence the thin-layer scalings yield the requirement that $u_{n}=u_{n}(x, t)$ must be independent of $\hat{y}$. It follows that $\hat{v}_{n}$ varies linearly between its values on the $n$th and $(n-1)$ th body surface. Writing

$$
(H, u, p)=\left(f_{n}^{-}-f_{n-1}^{+}, u_{n}, p_{n}\right)
$$

for each gap, the equations of motion within the fluid gaps become

$$
\partial H / \partial t+\partial(u H) / \partial x=0,
$$




$$
\partial u / \partial t+u \partial u / \partial x=-\partial p / \partial x,
$$

i.e. the shallow-water equations, where the influence of the unknown gap width $\left(f_{n}^{-}-f_{n-1}^{+}\right)$shows up. The Euler jumps local to the leading edges also impose the constraints

$$
p+\frac{1}{2} u^{2}=\frac{1}{2} \quad \text { at } \quad x=0+
$$

in each gap whereas the Kutta conditions at the successive trailing edges yield

$$
\text { each } p=\pi_{e}(t) \text { at } x=1-\text {, }
$$

for all $n$ with the unknown downstream pressure level $\pi_{e}(t)$ being independent of $n$. If for convenience we also define $f_{0}^{+}=0, f_{N}^{-}=1$ for the containing wall surfaces at all $t$ then the overall mass-conservation balance requires

$$
\sum_{1}^{N} u_{n}\left(f_{n}^{-}-f_{n-1}^{+}\right)=1 \quad \text { at } \quad x=1-,
$$

in view of the incident conditions ahead of the array of bodies. The above equations (4a-4e) describe the fluid-dynamical part of the interactive motion.

In the solid-body part of the motion each body is driven predominantly here by the fluid-dynamical pressure forces acting laterally on either of its surfaces. At this stage it is useful to be more explicit about the bodies, allowing for fixed shapes of overbody and underbody $F_{n}^{+}(x), F_{n}^{-}(x)$ respectively and thus thickness and camber in general. The body surfaces can then be specified simply by

$$
f_{n}^{ \pm}(x, t)=F_{n}^{ \pm}(x)+h_{n}(t)+\left(x-\frac{1}{2}\right) \theta_{n}(t),
$$

with $h_{n}, \theta_{n}$ giving the midpoint of the $n$th body and the body's angle of inclination in turn and being unknown functions of time $t$; see figure 1 again. The midpoint positions are the centres of mass for bodies which as here have uniform density distribution. In consequence the equation of lateral motion for each body takes the form

$$
M_{n} \frac{d^{2} h_{n}}{d t^{2}}=\int_{0}^{1}\left(p_{n}-p_{n+1}\right) d x .
$$

Here $M_{n}$ is the scaled mass of the $n$th body per unit width normal to the $x$ - $y$ plane, given by $M_{n} \equiv M_{n}^{*} \beta /\left(\rho^{*} L_{1}^{* 2}\right)$ where the dimensional body mass $M_{n}^{*}$ might vary over the $(N-1)$ bodies and the relations $(1 \mathrm{a}, 1 \mathrm{~b})$ are taken into account. Similarly the equation of angular motion of each body gives

$$
I_{n} \frac{d^{2} \theta_{n}}{d t^{2}}=\int_{0}^{1}\left(x-\frac{1}{2}\right)\left(p_{n}-p_{n+1}\right) d x
$$

where the scaled moment of inertia $I_{n} \equiv I_{n}^{*} \beta /\left(\rho^{*} L_{1}^{* 4}\right)$ and $I_{n}^{*}$ is the dimensional moment of inertia of the $n$th body. The central case of $\left(M_{n}, I_{n}\right)=(M, I)$ being independent of $n$ will be our concern here. The equation of axial bodymotion simply confirms that the incident flow velocity, and the location $x_{0}^{*}$, 
remain constant over the current scales because the axial forces on the body are relatively small.

The system controlling the fluid-body interactions is (4a-4e), (5a-5c) subject to suitable initial conditions, with $n$ running from 1 to $N$ (the number of gaps) in (4a-4d) and from 1 to $(N-1)$ (the number of bodies) in (5a-5c). The unknown fluid pressures and axial velocities $p_{n}, u_{n}$ for $n=1$ to $N$ depend on $x, t$, and the trailing edge pressure $\pi_{e}(t)$ is also unknown at each time $t$, while the unknown body midpoint positions and angles $h_{n}, \theta_{n}$ respectively for $n=1$ to $(N-1)$ also depend on the scaled time $t$ alone.

Analytical and computational properties are of interest. Numerical results from a finite-difference approach are given in [1] for a variety of $N$ values and in [3],[10] for various body shapes. Clashes are commonly indicated. Analytically the characteristic clash occurs through a nonlinear solution structure in which the evolving gap width $H(x, t)$ tends to zero at one particular station $x$ in one particular gap within a finite time. The clash at a leading edge has the contributions $d h / d t, d \theta / d t$ to the body velocity acquiring the form

$$
\begin{gathered}
\left(\frac{d h}{d t}, \frac{d \theta}{d t}\right)=\left(a_{1}, b_{1}\right)+B(t)\left(a_{2}, b_{2}\right)+\ldots, \\
\text { where } B(t)=-\left\{\ln \left(t_{0}-t\right)\right\}^{-1}+O(1),
\end{gathered}
$$

which occurs commonly for thin bodies, as $t \rightarrow t_{0}-$. In (6a, 6b) the constants $a_{1}, b_{1}, a_{2}, b_{2}$ are $O(1)$, while the irregular behaviour in $B(t)$ implies that the body acceleration becomes large along with the induced pressures locally. A corresponding feature is observed in the numerical results. For thicker bodies the clash occurs in a different form [3], at a station between the leading and trailing edges. Analysis also establishes the large- $N$ form of the system and, for any $N$ value, linear instability of the uniform state with growth rates that are found to agree [1] with the numerical results.

\section{$2.2 \quad$ A skimming body}

The same governing equations $(4 \mathrm{a}, 4 \mathrm{~b})$ apply in the water flow beneath a skimming body on a shallow water layer [2]: see figure 2. As the depth $H(x, t)$ evolves during a typical skimming event an analogue of (4c) due to [39] holds at the unknown leading contact point $x=x_{1}(t)$, while (4d) is replaced by $p=p_{0}$ at $x=1$ - where $p_{0}$ is the atmospheric pressure in the air above the water and the trailing edge is assumed sharp, giving a fixed trailing contact point. A free surface evolves in the wake downstream and a splash jet and free surface upstream. The body-motion equations are as in $(5 \mathrm{a}-5 \mathrm{c})$ without the superscripts $( \pm)$ and without subscripts $n$, with $p_{n+1}$ replaced by $p_{0}$ since the air flow is assumed dynamically negligible, and with the integration range being $\left(x_{1}, 1\right)$ which covers the wetted portion of the body.

Solutions are presented in [2], [5], [9] for thin bodies with $x_{1}$ at $1-$ and velocity $d x_{1} / d t$ negative at entry. See also figure 2. Depending on the initial conditions the body eventually either becomes totally submerged (when $x_{1}$ becomes less 


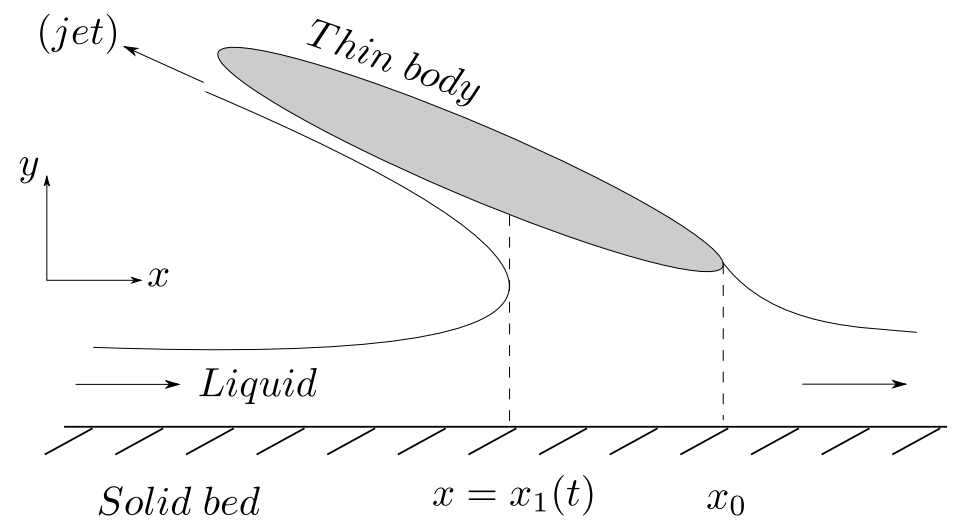

Fig. 2. Thin body skimming over a shallow liquid layer. Arrows indicate the direction of liquid motion in a frame of reference in which the solid body does not appear to move horizontally. The leading-edge position (i.e. contact point) $x_{1}$ varies with time $t$ whereas the trailing edge $x_{0}$ is fixed.

than the fixed leading edge location, $x=0$ say, of the body) or continues to skim off by emerging from the water when $x_{1}$ becomes 1 - after a finite time. The onset of this emergence involves a response in which a behaviour very akin to that in $(6 \mathrm{a}, 6 \mathrm{~b})$ recurs.

Recent work [8], [9] applies allied ideas to the sinking of the body where the upper surface becomes wetted. The body re-emerges through the free surface in some cases but otherwise usually hits the solid bed under the water layer within finite time in a clashing process that is focussed near the trailing edge.

\subsection{Pre-liftoff of a body from a solid surface}

Here a body is initially at rest or rocking back and forth on a flat horizontal surface or ground. A uniform horizontal flow of fluid is then started impulsively as in figure 3. Will the body continue to rock on the surface or instead tend to lift off? This question is addressed [4] by means of (4a, 4b) holding in the two thin gaps of evolving thickness $H(x, t)$ between the underbody and the ground, one gap upstream on the left and one downstream on the right of the moving contact point $x=x_{c}(t)$. The body itself is not necessarily thin. Condition (4c) applies at the left end of the left gap and $(4 \mathrm{~d})$ with $\pi_{e}(t)$ atmospheric $\left(p_{0}\right)$ at the right end of the right gap, whereas a consistency constraint applies at the unknown position $x=x_{1}(t)$. With affixes omitted the form (5a) describes $H$ in terms of underbody shape $F(x)$, height factor $h$, rotation angle $\theta$ analogously and $(5 \mathrm{~b}, 5 \mathrm{c})$ describe the underbody motion where $p_{n+1}$ is $p_{0}$ and in $(5 \mathrm{~b}) \mathrm{a}$ scaled normal reaction $R(t)$ and weight $M g$ are added in.

Properties from analysis and computation [4] show several interesting responses (continued rock or finite-time liftoff where $R(t)$ goes to zero or imme- 


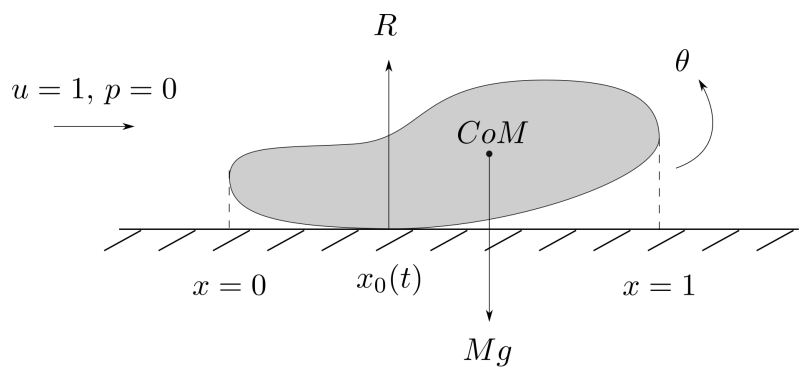

Fig. 3. Sketch showing two thin fluid layers, one for $0<x<x_{0}(t)$, the other for $x_{0}(t)<x<1$, beneath a body rocking on the ground with oncoming flow. The contact point $x_{0}(t)$, the reaction force $R(t)$ and the rotation angle $\theta(t)$ vary with time $t, M g$ is the weight, and $C o M$ is the centre of mass.

diate liftoff being indicated). Further studies have been made in recent Masters projects at UCL.

\subsection{Viscous-inviscid effects}

The interaction structure is more complicated when significant viscous effects are present. A central example has a thin body of length comparable with the width of the channel containing it [6], the body being nearly aligned with the oncoming non-uniform fluid flow which is a planar Poiseuille or similar flow $u\left(=u^{*} / U^{*}\right)=u_{0}(y)$. See figure 4 . The dimensional length $L_{1}^{*}$ is now the channel width. Over the axial scale $x$ of $O(1)$ we are led to linearised Euler flow past the body in the form

$$
u_{0}(y)\left(\frac{\partial}{\partial x^{2}}+\frac{\partial}{\partial y^{2}}\right) \psi_{1}=u_{0}^{\prime \prime}(y) \psi_{1},
$$

since the vorticity is nonzero, where $\psi_{1}$ is the scaled perturbation stream function. The major boundary conditions with $L$ standing for the non-dimensional length of the body are

$$
\psi_{1}=0 \quad \text { at } \quad y=0,1,
$$

no exponential growth far upstream and far downstream,

$$
\psi_{1}^{ \pm}=u_{0}\left(y_{0}\right)\left(-f^{ \pm}+K^{ \pm}\right) \quad \text { at } \quad y=y_{0}^{ \pm} \quad \text { for } \quad 0<x<L,
$$

for reasons of tangential flow at the walls, matching upstream and downstream and tangential flow on the overbody and underbody surfaces respectively. The latter surfaces are given effectively by (5a), without subscripts, whereas $K^{ \pm}$are unknown functions of scaled time $T$ only. At the channel walls $y=0,1$ there are thin passive viscous layers to reduce the flow velocity to zero at the walls and likewise for viscous layers on the body. It is assumed that the time scale of the body movements discussed below is much larger than the usual flow time scales and dominates the interactions here leaving the flow as quasi-steady. Also the solution is not defined uniquely by the system (7a-7d), an important issue here 


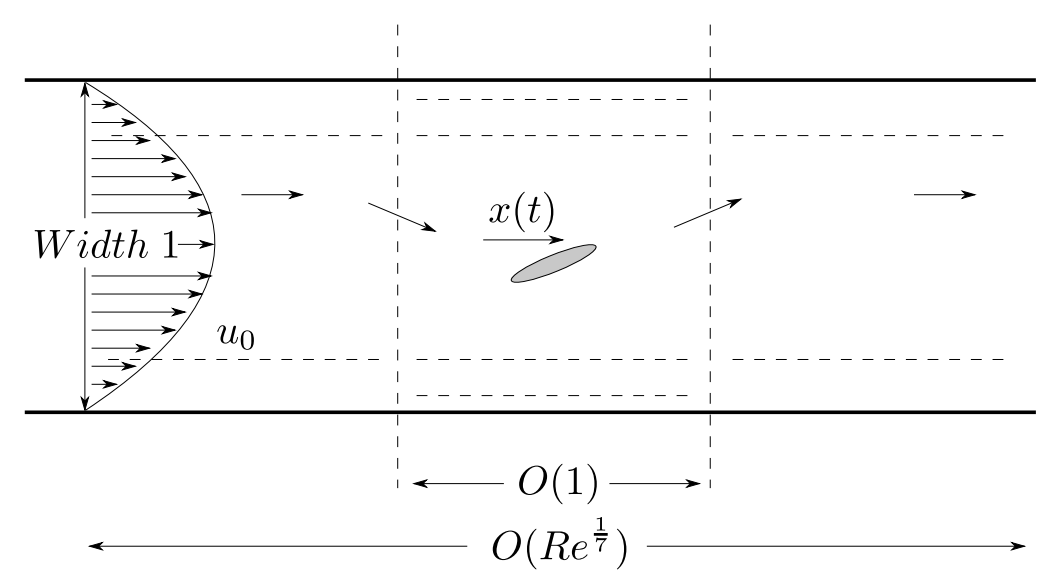

Fig. 4. A freely moving body in channel flow with vorticity [6]. Here the position $x(t)$ is almost constant and the single short arrows indicate a typical particle path in the core of the fluid flow.

owing to the fact that arbitrary multiples of $u_{0}(y)$ and $x u_{0}(y)$ can be added to any solution $\psi_{1}$ and still leave the system satisfied.

To resolve the issue the behaviour over a longer length scale $x=O\left(R e^{1 / 7}\right)$ needs accounting for, where $R e$ is based now on the channel width. An expansion analogous to (1b) holds in the inviscid core flow then for $0<y<1$ but with only small perturbations around $u=u_{0}(y)$ which produce an unknown displacement as well as a nonzero normal pressure gradient instead of (2c). A different expansion then holds in each viscous wall layer, such that each layer is controlled by the boundary layer equations

$$
\begin{gathered}
\partial U / \partial X+\partial V / \partial Y=0 \\
U \partial U / \partial X+V \partial U / \partial Y=-\partial P(X, T) / \partial X+\partial^{2} U / \partial Y^{2},
\end{gathered}
$$

where in particular $u=R e^{-2 / 7} U(X, Y, T), X=x / R e^{1 / 7}, Y=y R e^{2 / 7}$ in the lower layer, essentially the same in the upper layer, and the time scale $T$ is described below. The main constraints on $(8 \mathrm{a}, 8 \mathrm{~b})$ require [40] zero slip at $Y=0$, matching with the displaced core at large positive $Y$, satisfying a wall-pressuredifference condition between the two viscous layers, boundedness as $X \rightarrow \pm \infty$ and matching with the linearised Euler flow of (7a-7d) as $X \rightarrow 0 \pm$ since the body and its nearby flow appear as a point disturbance on the longer length scale. Details given in [6] show how the nonlinear viscous-inviscid system associated with $(8 \mathrm{a}, 8 \mathrm{~b})$ acts to determine the correct multiples of $u_{0}(y)$ and $x u_{0}(y)$ in the near-body system of (7a-7d).

Coupled with $(7 \mathrm{a}-7 \mathrm{~d})$ are the evolution equations for the movement of the body which are essentially as in (5a-5c) in altered notation. Solutions are presented in [6]. A common finding is instability over the present time scales such that the small angle $\theta$ increases exponentially with increasing time $T$. 
Another central case has a thin body of length almost comparable with the development length of the channel flow or boundary layer [7]; in the boundary layer setting on an airfoil the development length is usually a significant fraction of the airfoil chord. This case examines a direct link between the moving body shape, acting as a displacement, and the pressure response $P$ in the wall layer(s). The response $P$ then affects the body movement as in $(5 \mathrm{a}-5 \mathrm{c})$, leading once more to dynamic fluid-body interaction. A new form of instability is again found unless mitigation measures are taken [7].

The findings and ideas summarised in sections 2.1-2.4 above provide the basis for the more recent and continuing studies considered in the following three sections.

\section{Skimming by a smooth body}

The skimming process for a smoothly curved body in general can be decomposed into two consecutive stages [5], [41], [42]: an initial impact stage followed by a planing stage, after which the body either sinks or lifts off from water and thereby completes one skipping (skimming) cycle. We present a shallow water impact and planing model, and then investigate how such a smooth body is able to rapidly transit from its initial impact to a planing motion. We shall also analyse how the presence of an adverse pressure gradient in the trailing separation flow, which is typically associated with flows past a smooth or bluff body, can affect its planing motion.

The skimming object of our interest here has an elongated horizontal profile, whose length $\bar{l}$ is much greater than its thickness; its lower body surface, which may be in contact with water, is smooth and strictly convex. Suppose $\bar{h}$ is the depth of the water: its shallowness implies $\bar{h} \sim \epsilon \bar{l}$ with parameter $\epsilon \ll 1$. This object could be skimming at an inclined angle, say $\alpha$, as well as having an angular velocity $\omega$; the angle of inclination, defined as the one made by its major axis and the undisturbed water free surface, is assumed to be small such that $\alpha \sim O(\epsilon)$. Letting $(\bar{u}, \bar{v})$ be the body's horizontal and vertical velocities respectively, in our model of interest the horizontal speed is a magnitude larger than the vertical speed, i.e. $\bar{v} \sim \epsilon \bar{u}$. See figure 5 for a depiction of the coordinate system and necessary nomenclature.

An upper-half Cartesian coordinate system $(\bar{x}, \bar{y})$ is introduced such that its $\bar{x}$-axis rests at the bottom of the shallow water and points in the opposite direction to the skimming body's horizontal velocity $\bar{u}$. Its $\bar{y}$-axis points upwards and goes through the skimming body's centre of mass, whose coordinate is $\left(0, \bar{y}_{m}\right)$. In this configuration the coordinate system travels horizontally with the skimming body, the undisturbed water flows in the positive direction of the $\bar{x}$-axis and the body itself has only vertical and angular motions.

As the body impacts on water, the flow can be approximately divided into three sub-regions: an undisturbed far-stream flow region, where the water is undisturbed and at rest; a region with elevated free surface of size $O(\bar{h}) \times O(\bar{h})$, known as the "jet-root" or "turn-over" region where the flow separates from the 


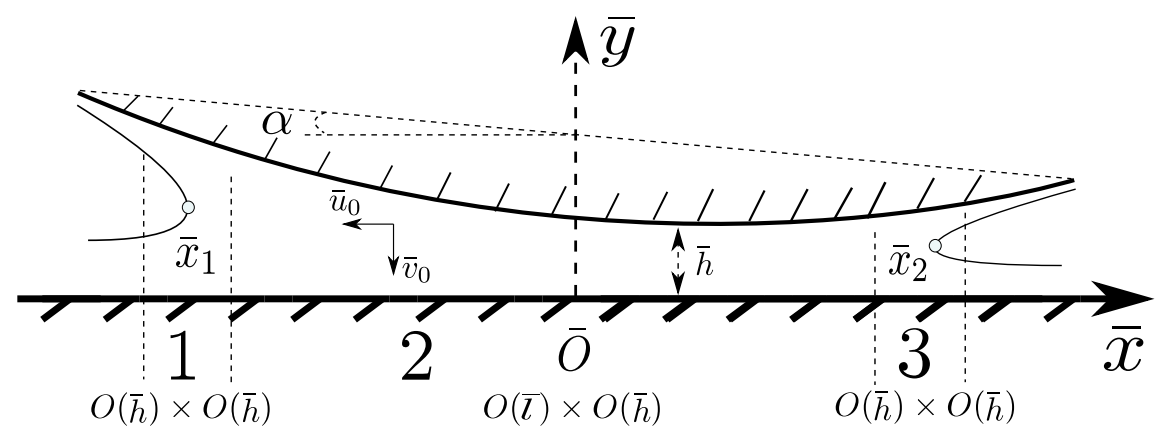

Fig. 5. A close-up sketch of a smoothly curved body during the impact stage of a skimming process. $\bar{x}_{1}$ and $\bar{x}_{2}$ represent the horizontal positions of the leading and trailing contact edges respectively. $\bar{h}$ denotes the representative depth of the water layer, the jet root regions 1 and 3 have representative scales of $O(\bar{h}) \times O(\bar{h})$, while the region underneath the impact body has size of $O(\bar{l}) \times O(\bar{h})$. At a sufficiently small time, the contact surface of the blunt body can be approximated by a flat plate at the leading order, and $\bar{x}_{1}, \bar{x}_{2}$ move away from each other at an extremely large speed.

body and splash jets may be emitted; and finally a main flow region trapped under the body and enclosed by the jet-root region. The splash jets are typically thin compared with the main body flow and their effects can be neglected [39], [43].

We let $\bar{x}_{1}, \bar{x}_{2}$ be the horizontal positions (called here the leading edge and trailing edge respectively) of the stream turn-over points in the jet-root region, which are not known a priori and would need to be found as part of the skimming system. Once the leading and trailing edge positions are determined together with the known skimming body's surface function, the total contact area between the body and the fluid can be determined.

\subsection{Impact model}

Taking $\left(\bar{u}_{0}, \bar{v}_{0}\right)$ to be the body's initial horizontal and vertical velocities respectively, the system can be non-dimensionalized as follows:

$x=\frac{\bar{x}}{\bar{l}}, \quad y=\frac{\bar{y}}{\bar{l}}, \quad h=\frac{\bar{h}}{\bar{l}}, \quad t=\frac{\bar{l}}{\bar{u}_{0}}, \quad \tilde{u}=\frac{\bar{u}}{\bar{u}_{0}}, \quad \tilde{v}=\frac{\bar{v}}{\bar{u}_{0}}, \quad \tilde{p}=\frac{\bar{p}}{\rho \bar{u}_{0}^{2}}, \quad m=\frac{\bar{m}}{\rho \bar{h} \bar{l}}, \quad i=\rho \bar{l}^{4} \bar{i} ;$

where $\bar{m}$ and $\bar{i}$ are the body's mass and moment of inertia respectively.

Suppose the body's lower surface is given by $\eta(x)$, which is smooth and strictly convex, and can be expressed locally in a parabolic form:

$$
\eta(x)=a x^{2}+b x+c \quad(\text { where } a<0) .
$$


The coefficients $a, b, c$ are constants that can be calibrated according to the object's shape. Notice that to ensure our skimming body has a horizontally elongated profile, the surface coefficients are small: $a, b, c \sim O(\epsilon)$. The water depth under the body can be obtained:

$$
h(x, \alpha, t)=y_{m}(t)+x \alpha(t)-\eta(x), \quad\left(x \in\left[x_{1}(t), x_{2}(t)\right]\right) .
$$

The large differences between the horizontal and vertical scales of our problem can be exploited by introducing the expansion:

$(\tilde{u}, \tilde{v}, \tilde{p}, y, h, a, b, c, \alpha, \eta) \sim(1,0,0,0,0,0,0,0,0,0)+\epsilon(u, V, p, Y, H, A, B, C, \theta, T)$,

where the newly scaled variables on the right-hand-side are of order unity and $\epsilon=\bar{h}_{0} / \bar{l} \ll 1$ as before. The water depth and the body surface equations now become:

$$
\begin{array}{lr}
H(x, \theta, t)=Y_{m}(t)+x \theta(t)-T(x), & \left(x \in\left[x_{1}(t), x_{2}(t)\right]\right) ; \\
T(x)=A x^{2}+B x+C, & (A<0) .
\end{array}
$$

For a body skimming at a sufficiently large horizontal velocity, a straightforward scaling analysis (see [2], [5], [39] for example) shows that pressure force dominates over other effects such as viscosity, gravity and surface tension in the flow. Therefore the Navier-Stokes equations at the leading order can be reduced to:

$$
\begin{aligned}
& \frac{\partial u}{\partial t}+\frac{\partial u}{\partial x}=-\frac{\partial p}{\partial x} \\
& \frac{\partial p}{\partial Y}=0
\end{aligned}
$$

Applying the kinematic boundary condition at the surface of the flow yields this additional relation:

$$
\frac{\partial H}{\partial t}+\frac{\partial H}{\partial x}+\frac{\partial u}{\partial x}=0
$$

By Newton's third law of reciprocity the skimming body's vertical and angular momentum equations at the leading order can be written as:

$$
\begin{aligned}
& \int_{x_{1}}^{x_{2}} p(x, t) d s=M \frac{d^{2} Y_{m}}{d t^{2}}, \\
& \int_{x_{1}}^{x_{2}} x p(x, t) d s=I \frac{d^{2} \theta}{d t^{2}},
\end{aligned}
$$

where $M=\epsilon m, I=\epsilon i$. Notice the horizontal force on the body is only of order $\epsilon$, so that in the time scale of our consideration the skimming horizontal velocity is unchanged at the leading order.

Imposing the pressure jump conditions as introduced by [2], [39], [42], [44] together with Bernoulli's principle at the leading and trailing edges we derive 
the boundary conditions for our impact problem as follows:

$$
\begin{aligned}
& p\left(x_{1}, t\right)+\frac{1}{2}\left(u\left(x_{1}, t\right)-\frac{d x_{1}}{d t}\right)^{2}=\frac{1}{2}\left(1-\frac{d x_{1}}{d t}\right)^{2}, \\
& p\left(x_{2}, t\right)+\frac{1}{2}\left(u\left(x_{2}, t\right)-\frac{d x_{2}}{d t}\right)^{2}=\frac{1}{2}\left(1-\frac{d x_{2}}{d t}\right)^{2}, \\
& \left(u\left(x_{1}, t\right)-\frac{d x_{1}}{d t}\right) /\left(1-\frac{d x_{1}}{d t}\right)=2 H\left(x_{1}, t\right)^{-\frac{1}{2}}-1, \\
& \left(u\left(x_{2}, t\right)-\frac{d x_{2}}{d t}\right) /\left(1-\frac{d x_{2}}{d t}\right)=2 H\left(x_{2}, t\right)^{-\frac{1}{2}}-1 .
\end{aligned}
$$

Our impact model therefore consists of (12) - (16).

We focus on a linearised formulation of this integro-differential problem in the next subsection to gain further insights.

\subsection{Linearised impact model and rapid transition to planing motion}

During the impact phase the solid-liquid contact surface initially goes through a phase of rapid expansion and spray jets are formed at the boundary of the surface. To analyse the impact system behaviour during this phase of rapid contact surface expansion, we focus on a short time after impact such that time $t$ is of order $\delta$ where $\delta \ll 1$.

Given that the body's initial vertical velocity is of order unity, we expect the free surface penetration to be small and have the same order as time $t$. Balancing the terms of the free surface equation (12a) suggests that the model's horizontal and angular scales both evolve on a higher order, specifically $x \sim O\left(\delta^{\frac{1}{2}}\right)$ and $\theta \sim O\left(\delta^{\frac{1}{2}}\right)$. From the pressure jump conditions (16a) and (16b) we can deduce that the fluid's horizontal velocity $u$ evolves on the same scale as $x$ and that the pressure $p$ evolves on the scale of order unity. We therefore asymptotically expand the system variables as follows:

$$
\begin{aligned}
& t=\delta \hat{t}, \\
& x \sim x_{0}+\delta^{\frac{1}{2}} \hat{x}+O(\delta), \\
& Y_{m} \sim Y_{0}+\delta \hat{Y}+O\left(\delta^{2}\right), \\
& \theta \sim \theta_{0}+\delta^{\frac{1}{2}} \hat{\theta}+O(\delta), \\
& H \sim 1+\delta \hat{H}+O\left(\delta^{2}\right), \\
& u \sim \delta^{\frac{1}{2}} \hat{u}+O(\delta), \\
& p \sim \hat{p}+O\left(\delta^{\frac{1}{2}}\right) .
\end{aligned}
$$

Applying asymptotic analysis of such form to our impact model indicates that, unless the body has sufficiently small body mass $\left(M \sim O\left(\delta^{\frac{3}{2}}\right)\right)$, the force generated by hydraulic pressure underneath the body does not have a significant 
effect on the body's momentum in a short time after impact. At the leading order we thus expect the body's vertical and angular velocities to be equal to their initial values: $d \hat{Y} / d \hat{t} \sim \hat{V}_{0}$ and $d \hat{\theta} / d \hat{t} \sim \hat{\omega}_{0}$.

If on the other hand our skimming object has a small mass, for instance $M \sim$ $O\left(\delta^{\frac{3}{2}}\right)$, then its vertical momentum equation (15a) has an immediate response to the flow pressure even inside our small time regime in (17). The linearised impact system takes on a form of five differential algebraic equations (DAEs) for five unknowns: $\hat{x}_{1}, \hat{x}_{2}, \hat{Y}, \hat{f}$ and $\hat{g}$ :

$$
\begin{aligned}
& \hat{Y}=\frac{1}{3} A\left(\hat{x}_{1}^{2}+\hat{x}_{1} \hat{x}_{2}+\hat{x}_{2}^{2}\right)-\frac{1}{2} \hat{\omega}_{0}\left(\hat{x}_{1}+\hat{x}_{2}\right) \hat{t}, \\
& \hat{f}=-\frac{1}{6} \hat{\omega}_{0}\left(\hat{x}_{1}^{2}+\hat{x}_{1} \hat{x}_{2}+\hat{x}_{2}^{2}\right)-\frac{1}{2}\left(\hat{x}_{1}+\hat{x}_{2}\right) \frac{d \hat{Y}}{d \hat{t}}, \\
& \hat{f}=\frac{1}{2} \frac{d}{d \hat{t}}\left[\frac{A}{3}\left(\hat{x}_{1}^{3}+\hat{x}_{2}^{3}\right)-\frac{\hat{\omega}_{0}}{2}\left(\hat{x}_{1}^{2}+\hat{x}_{2}^{2}\right) \hat{t}-\left(\hat{x}_{1}+\hat{x}_{2}\right) \hat{Y}\right], \\
& \hat{g}=-\frac{1}{2} \frac{d}{d \hat{t}}\left[\frac{\hat{\omega}_{0}}{6}\left(\hat{x}_{1}^{3}+\hat{x}_{2}^{3}\right)+\frac{1}{2}\left(\hat{x}_{1}^{2}+\hat{x}_{2}^{2}\right) \frac{d \hat{Y}}{d \hat{t}}+\left(\hat{x}_{1}+\hat{x}_{2}\right) \hat{f}\right], \\
& \left(6 \hat{M}+\hat{x}_{1}^{3}-\hat{x}_{2}^{3}\right) \frac{d^{2} \hat{Y}}{d \hat{t}^{2}}+3\left(\hat{x}_{1}^{2}-\hat{x}_{2}^{2}\right) \frac{d \hat{f}}{d \hat{t}}+6\left(\hat{x}_{1}-\hat{x}_{2}\right) \hat{g}=0,
\end{aligned}
$$

where $\hat{M}=\delta^{\frac{3}{2}} M$.

Numerical analysis of this DAEs system indicates that for a body with positive/forward rotation (i.e. $\hat{\omega}_{0}>0$ ), a retraction of the trailing edge position could occur inside this small-time regime, see figure 6 for a demonstration.

It is seen that at the instant of touchdown the speeds at which the leading and trailing edges evolve away from the initial contact point are very large, hence the wetted surface expands rapidly immediately after impact. The "outward expansion" speeds of the two edges decrease as time progresses however, and for a forward-rotating body the trailing edge's velocity eventually drops to zero (figure 6b).

Letting $\hat{t}_{c}$ denote the critical time when this phenomenon occurs, before this critical time is reached the fluid is thrown away from the skimming body's leading edge towards upstream, which is signified by the fluid velocity being negative at this edge as demonstrated in figure 6c. At the trailing edge the fluid velocity is initially positive, but as the critical time is approached this gradually decreases to zero, which also corresponds to the trailing edge pressure decreasing to be atmospheric, see figure $6 \mathrm{~d}$. The vertical centre of mass position $\hat{Y}$ reaches its minimum shortly before this critical time and begins to move upwards. Hence before the trailing edge pressure drops to be atmospheric the body is already in the early stages of moving upwards in the water. It is evident therefore that the body mass and its angular rotation have an important and immediate effect, influencing the development of the wetted surface and thereby affecting the lift force on the body.

The pressure in the fluid is initially high and positive everywhere. In the case of a positive rotation the leading edge separation point sustains the highest 


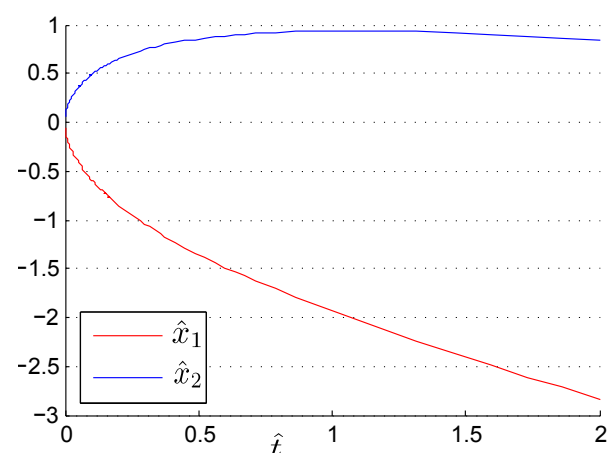

(a)

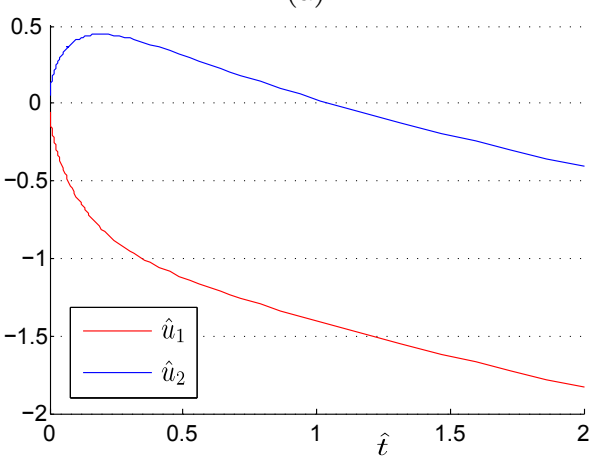

(c)

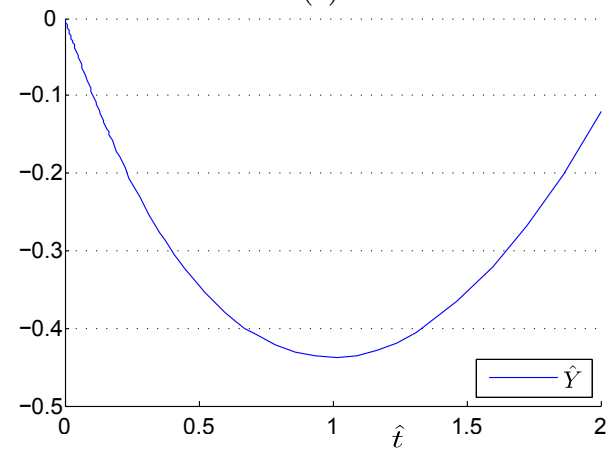

(e)

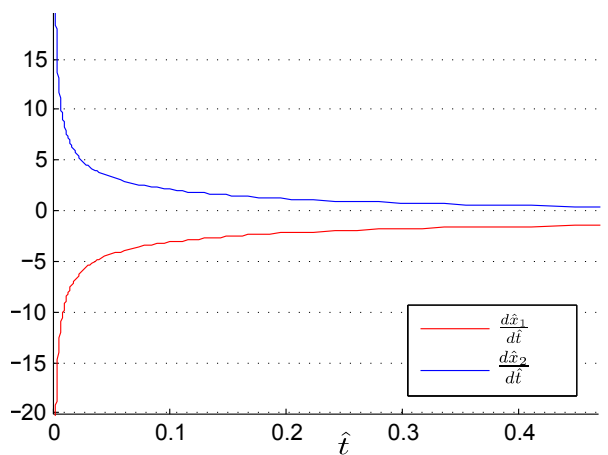

(b)

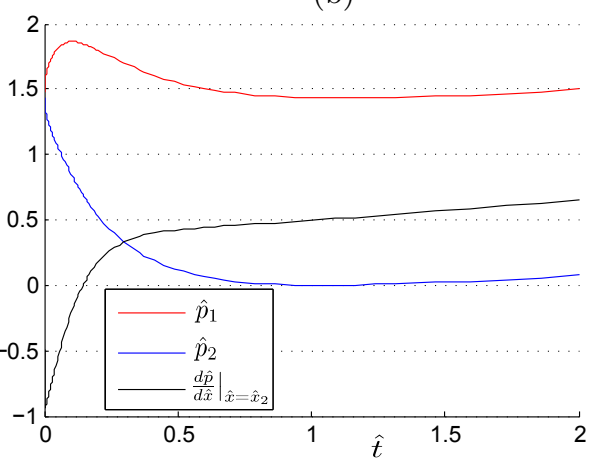

(d)

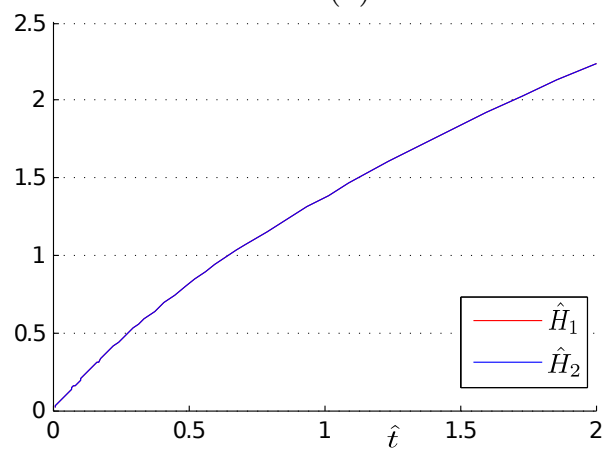

(f)

Fig. 6. Various profile plots of a skimming body with a positive rotation $\hat{\omega}_{0}>0$. The initial vertical velocity of the body $\hat{V}_{0}$ is taken to be -1 , and the initial angular velocity $\hat{\omega}_{0}$ is taken to be 1 . Under these initial settings the trailing edge $\hat{x}_{2}$ initially evolves towards the downstream, however at time $\hat{t} \sim 1.0497$ this edge reaches its maximum and begins to retract towards upstream initial contact point. (a) Evolution of leading and trailing edges $\hat{x}_{1}, \hat{x}_{2}$ w.r.t. time $\hat{t}$. (b) Evolution of leading and trailing edge velocities $\frac{d \hat{x}_{1}}{d \hat{t}}, \frac{d \hat{x}_{2}}{d \hat{t}}$. (c) The evolution of fluid velocities $\hat{u}_{1}$ and $\hat{u}_{2}$. (d) Evolution of leading and trailing edge pressure profiles. (e) Vertical evolution of the skimming body's centre of mass. (f) Water surface elevation at the leading and trailing edges. 


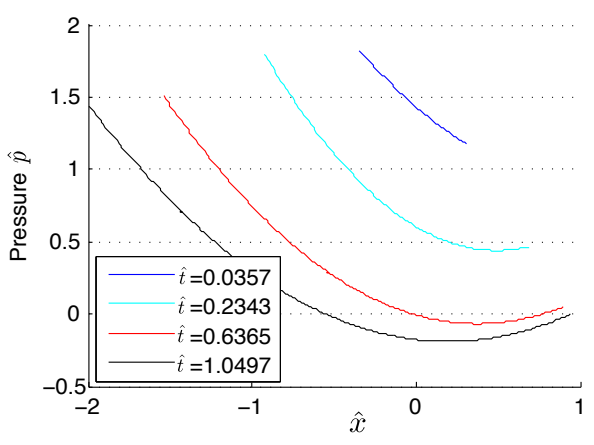

(a)

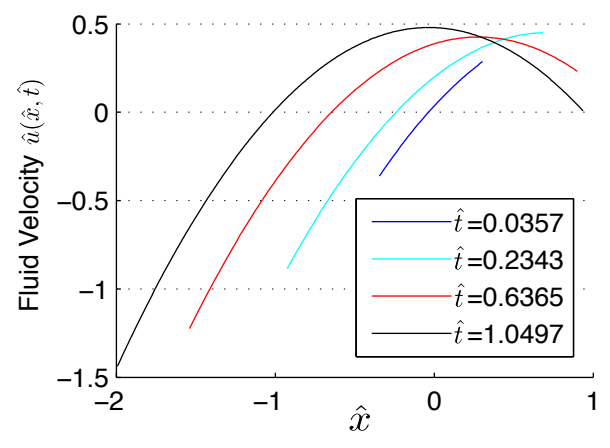

(b)

Fig. 7. The time evolution of the pressure and fluid velocity underneath the skimming body during the impact stage for a positively rotating body. The time begins from shortly after impact $\hat{t}=0.0357$ to the end of impact stage at $\hat{t}=1.0497$ when the trailing edge pressure drops to zero. Notice that the wetted surface area is initially small and increases over time. The initial conditions are $\hat{V}_{0}=-1$ and $\hat{\omega}_{0}=1$. (a) A plot of pressure profiles $\hat{p}$ underneath the skimming body at different times of the impact stage. (b) A plot of fluid velocity profiles $\hat{u}$ underneath the skimming body at different times of the impact stage.

pressure, as is demonstrated in figure 7a. As time progresses the contact surface grows and pressure decreases everywhere; eventually a negative pressure region begins to develop in a region close to the body's trailing edge, and in particular an adverse pressure gradient field inside this negative pressure region begins to form. This sub-atmospheric pressure region eventually reaches the trailing edge, at which point the trailing edge fluid velocity drops to zero, i.e. water is no longer ejected away at the trailing edge: see figure $7 \mathrm{~b}$ for an illustration.

Once this critical time is reached the skimming body changes from its initial impact phase to a planing phase. In the next subsection we describe the planingphase model.

\subsection{Planing model}

The change from impact to planing stage is marked by the position of the trailing edge which stops extending further towards the downstream direction, as well as the disappearance of the spray jet at this edge. Our analysis in Section 3.2 shows that a region with negative pressure gradient begins to develop near the trailing edge at the end of the impact stage. This phenomenon is consistent with the separation of a high-Reynolds-number incompressible flow passing a bluff body.

For the case of a completely submersed body, the presence of a significant adverse pressure gradient field causes the flow to separate from the body, a behaviour which is well analysed using triple-deck theory (see [45]-[47]) for the laminar or turbulent regime. For the planing case where the body is only partially submersed in water, experiments show that in the region of separation near the 
trailing edge there is a turbulent mixture of air and water. One can speculate for continuity that there should be an adverse pressure gradient at the trailing separation edge, at least for a brief period of time, when the body switches from impact to planing motion. Such an adverse pressure gradient, say $\kappa$ (or $\kappa(t)$ as a function of time), however, can only be determined via appropriate boundary layer analysis and that is considered beyond the scope of this discussion. A more detailed discussion can be found in reference [47]. Thus under the assumptions of turbulent separation the trailing edge boundary conditions can be prescribed as:

$$
p\left(x_{2}, t\right)=0,\left.\quad \frac{\partial p}{\partial x}\right|_{x=x_{2}}=\kappa .
$$

The governing equations for the fluid flow and the planing body then are very similar to those of the impact model, the differences being that: 1) in our planing model the time $t$ starts at $t=t^{*}$, where $t^{*}$ is the time at the end of the impact stage; and 2) at the trailing edge the momentum and pressure jump conditions are replaced by the separation conditions of (19).

In the sections that follow we pick up our analysis for a light body in Section 3.2 and investigate the planing behaviour.

\subsection{Linearised planing model}

Under the asymptotic settings of (17) our planing model in Section 3.3 reduces to a system of three ODEs with three unknowns $\hat{x}_{1}, \hat{x}_{2}$ and $\hat{Y}$ :

$$
\begin{aligned}
& {\left[6 \hat{M}+\left(\hat{x}_{1}-\hat{x}_{2}\right)^{3}\right] \frac{d^{2} \hat{Y}}{d \hat{t}^{2}}+3 \hat{\kappa}\left(\hat{x}_{1}-\hat{x}_{2}\right)^{2}=0} \\
& \frac{1}{2}\left(\hat{x}_{1}-\hat{x}_{2}\right)^{2} \frac{d^{2} \hat{Y}}{d \hat{t}^{2}}+\left(A \hat{x}_{1}^{2}-\hat{\omega}_{0} \hat{t} \hat{x}_{1}-\hat{Y}\right)\left(\frac{d \hat{x}_{1}}{d \hat{t}}\right)^{2}+\hat{\kappa}\left(\hat{x}_{1}-\hat{x}_{2}\right)=0 \\
& \left(\hat{x}_{1}-\hat{x}_{2}\right) \frac{d^{2} \hat{Y}}{d \hat{t}^{2}}-\left(A \hat{x}_{1}^{2}-\hat{\omega}_{0} \hat{t} \hat{x}_{1}-\hat{Y}\right) \frac{d^{2} \hat{x}_{1}}{d \hat{t}^{2}}-\left(2 A \hat{x}_{1}-\hat{\omega}_{0} \hat{t}\right)\left(\frac{d \hat{x}_{1}}{d \hat{t}}\right)^{2} \\
& \quad+2\left(\hat{\omega}_{0} \hat{x}_{1}+\frac{d \hat{Y}}{d \hat{t}}\right) \frac{d \hat{x}_{1}}{d \hat{t}}+\hat{\kappa}=0 .
\end{aligned}
$$

Here $\hat{\kappa}=\delta^{\frac{1}{2}} \kappa$ according to the scaling of (17).

In figure 8 we display solutions to our planing model for a range of prescribed $\hat{\kappa}$ values. It can be seen that the larger $\hat{\kappa}$ is, the further the flow is able to attach onto the body, which is represented by the position of $\hat{x}_{2}$, and therefore the greater the contact surface area between water and body. Further analysis (see [9]) reveals that there is a maximum adverse pressure gradient, say $\hat{\kappa}_{M}$, that can be sustained at the trailing edge, exceeding which our planing regime breaks down. Under the support of such a $\hat{\kappa}_{M}$ value the maximum distance between the leading and trailing edge positions can be deduced as follows:

$$
M A X\left(\hat{x}_{2}-\hat{x}_{1}\right)=[(24+18 \sqrt{2}) \hat{M}]^{\frac{1}{3}} .
$$




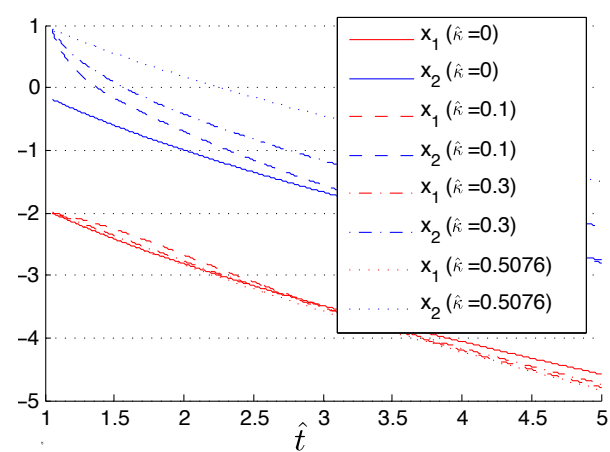

(a)

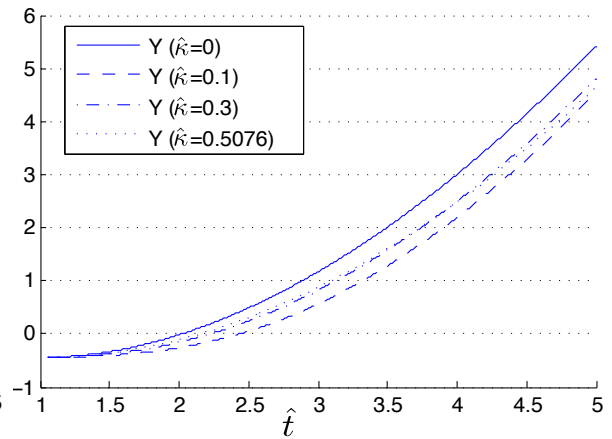

(b)

Fig. 8. Plot of the leading and trailing edges, as well as the plate's vertical centre of mass during the planing stage for varying values of trailing edge pressure gradient $\hat{\kappa}$. The body's mass $\hat{M}$ and rotational velocity $\hat{\omega}_{0}$ are both taken to be one. For the case of $\hat{\kappa}=0$ the trailing edge position $\hat{x}_{2}$ is not continuous when transition from impact to planing stage. (a) The evolution of the plate's leading and trailing edges during the planing stage. (b) The evolution of the plate's vertical centre of mass position during planing.

This limit is dependent only on the body mass, and intuitively the heavier the body, the further apart the leading and trailing edges can be.

If on the other hand the adverse pressure gradient is weak, $\hat{\kappa} \ll 1$, the body's planing motion can be divided into three distinct and consecutive phases, which are discussed next.

Initial planing phase. Let $\hat{t}_{c}$ denote the time when the body changes to the planing stage. We write $\hat{\xi}$ to be the horizontal distance between the leading and trailing edges: $\hat{\xi}=\hat{x}_{2}-\hat{x}_{1}$, and let $\hat{x}_{c 1}, \hat{Y}_{c}$ and $\hat{V}_{c}$ denote the horizontal position of the leading edge, vertical position of the centre of mass and vertical velocity respectively at time $\hat{t}_{c}$. Given that the pressure gradient satisfies $0<\hat{\kappa} \ll 1$, we postulate the following asymptotic expansions:

$$
\begin{aligned}
& \hat{x}_{1}=\hat{x}_{c 1}+\hat{\kappa}^{\frac{1}{2}} \check{x}_{1}(\hat{t})+O(\hat{\kappa}), \\
& \hat{\xi}=\check{\xi}_{1}(\hat{t})+O\left(\hat{\kappa}^{\frac{1}{2}}\right), \\
& \hat{Y}=\hat{Y}_{c}+\hat{V}_{c} \hat{t}+\hat{\kappa} \check{Y}_{1}(\hat{t})+O\left(\hat{\kappa}^{2}\right) .
\end{aligned}
$$


Our planing system can then be simplified to a system of differential algebraic equations of the following form:

$$
\begin{aligned}
& \frac{d}{d \hat{t}}\left[\zeta^{2} \frac{d \check{x}_{1}}{d \hat{t}}\right]=0 \\
& \check{\xi}_{1}^{4}+2 \zeta\left(6 M-\check{\xi}_{1}^{3}\right)\left(\frac{d \check{x}_{1}}{d \hat{t}}\right)^{2}+12 M \check{\xi}_{1}=0, \\
& {\left[6 M-\check{\xi}_{1}^{3}\right] \frac{d^{2} \check{Y}_{1}}{d t^{2}}+3 \check{\xi}_{1}^{2}=0}
\end{aligned}
$$

where the function $\zeta(\hat{t})$ is given as

$$
\zeta(\hat{t})=\hat{x}_{c 1}^{2}+\hat{Y}_{c}+\left(\hat{V}_{c}+\hat{\omega}_{0} \hat{x}_{c 1}\right) \hat{t} .
$$

Let $\check{Y}_{10}, \check{V}_{10}$ be the initial value and initial first order derivative for $\check{Y}_{1}$, and $\check{x}_{10}, \check{u}_{10}$ be the initial value and initial first order derivative for $\check{x}_{1}$ respectively. We may then apply the following Cauchy conditions:

$$
\begin{array}{ll}
\left.\check{Y}_{10} \equiv \check{Y}_{1}\right|_{\hat{t}=\hat{t}_{c}}=0, & \left.\check{V}_{10} \equiv \frac{d \check{Y}_{1}}{d \hat{t}}\right|_{\hat{t}=\hat{t}_{c}}=0 ; \\
\check{x}_{10}=0, & \check{u}_{10}=-\left[\frac{\check{\xi}_{10}^{4}+12 M \check{\xi}_{10}}{2 \zeta_{0}\left(\check{\xi}_{10}^{3}-6 M\right)}\right]^{\frac{1}{2}} .
\end{array}
$$

The initial condition for $\check{u}_{10}$ can be obtained by combining (23b, 23c) and setting $\hat{t}=\hat{t}_{c}$.

At this point we can write down the solution to $\check{x}_{1}$ :

$$
\check{x}_{1}(\hat{t})=\Phi_{0}\left(1-\zeta_{0} \zeta^{-1}\right),
$$

where $\Phi_{0}$ is given as

$$
\Phi_{0}=-\frac{\zeta_{0}}{\hat{V}_{c}+\hat{\omega}_{0} \hat{x}_{c 1}}\left[\frac{\check{\xi}_{10}^{4}+12 M \check{\xi}_{10}}{2 \zeta_{0}\left(\check{\xi}_{10}^{3}-6 M\right)}\right]^{\frac{1}{2}} .
$$

This explicit solution for $\check{x}_{1}$ leads to the following quartic equation of $\check{\xi}_{1}$ from (23b):

$$
\zeta^{3} \check{\xi}_{1}^{4}-2 \Phi_{0}^{2} \check{\xi}_{1}^{3}+12 M \zeta^{3} \check{\xi}_{1}+12 M \Phi_{0}^{2}=0 .
$$

The formula for finding roots of quartic equations is well known and will not be presented here explicitly. Out of the four possible solutions for $\check{\xi}_{1}$ the admissible one should be real, positive and fit the physical context of the system. From (23c) we can obtain the solution for $\check{Y}_{1}$ in a double integral form based on the admissible solution of $\check{\xi}_{1}$ and initial conditions (25a):

$$
\check{Y}_{1}=\iint_{\hat{t}_{c}}^{\hat{t}} \frac{3 \check{\xi}_{1}^{2}}{\check{\xi}_{1}^{3}-6 M} d \hat{t}^{2}
$$




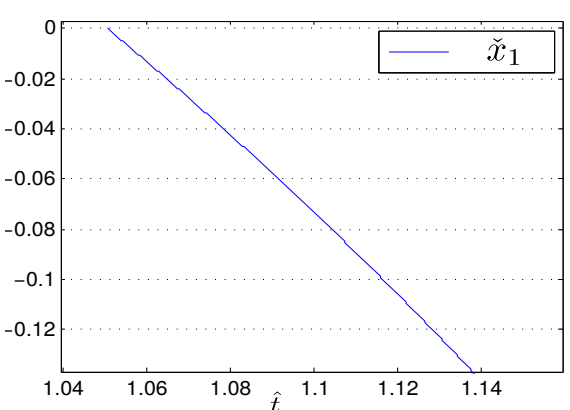

(a) $\check{x}_{1}$

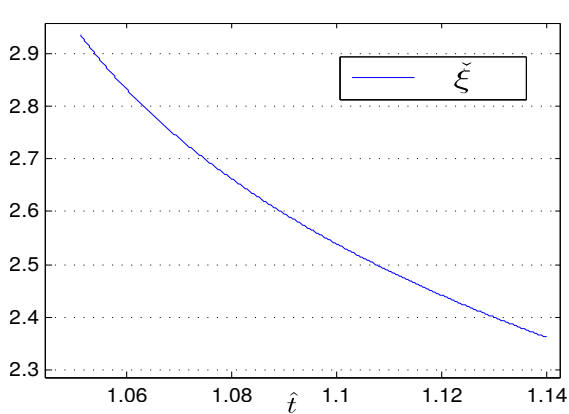

(b) $\check{\xi}_{1}$

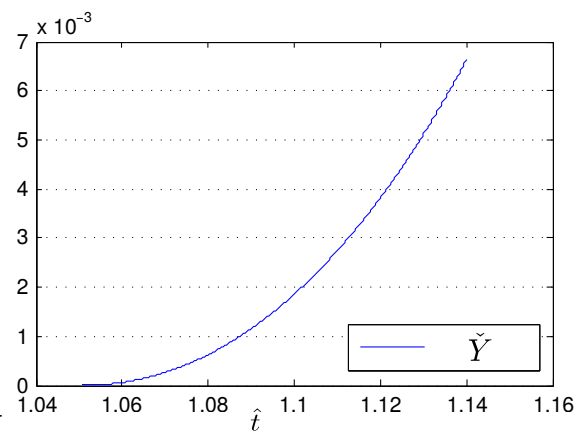

(c) $\check{Y}_{1}$

Fig. 9. Solutions of the planing system (23) with $M=1, \hat{\omega}_{0}=1, \hat{\kappa}=0.1$. The values of $\hat{x}_{c 1}, \hat{x}_{c 2}, \hat{Y}_{c}$ and $\hat{V}_{c}$ are the results from the final stage of impact model (18), their respective values are: $-1.9944,0.9434,-0.4431$ and 0.0359 .

The solutions for $\check{x}_{1}, \check{\xi}_{1}$ and $\check{Y}_{1}$ are presented in figure 9 . The results demonstrate that for a planing body with positive rotation, its leading edge position continues to extend in the upstream direction as with the impact stage case. The trailing edge position on the other hand also begins to move in the upstream direction, and it moves at a greater pace compared with that of the leading edge as demonstrated by the decreasing value of $\check{\xi}_{1}$ in figure $9 \mathrm{~b}$. This signifies that the contact surface between the water and planing starts to decrease. During this phase the planing body continues to emerge from the water as shown in figure $9 \mathrm{c}$.

Planing phase II. The solution of $\check{x}_{1}$ given in (26) depends inversely on $\zeta(\hat{t})$, which eventually decreases to zero as time progresses and $\check{x}_{1}$ becomes singular. Suppose $\hat{t}_{N}$ is the time when this singularity occurs. Then from the definition of $\zeta$ in $(24) \hat{t}_{N}$ can be deduced as:

$$
\hat{t}_{N}=-\frac{\hat{x}_{c 1}^{2}+\hat{Y}_{c}}{\hat{V}_{c}+\hat{\omega}_{0} \hat{x}_{c 1}}
$$

which is the time when the second phase of our planing stage begins. In this phase we seek a new asymptotic form as follows:

$$
\begin{aligned}
& \hat{t}=\hat{t}_{N}+\hat{\kappa}^{\frac{1}{4}} \bar{t} \\
& \hat{x}_{1}=\hat{x}_{c 1}+\hat{\kappa}^{\frac{1}{4}} \bar{x}_{1}+\ldots, \\
& \hat{\xi}=(6 M)^{\frac{1}{3}}-\hat{\kappa}^{\frac{3}{4}} \bar{y}_{1}+\ldots, \\
& \hat{Y}_{1}=\hat{Y}_{c}+\hat{V}_{c} \hat{t}_{N}+\hat{\kappa}^{\frac{1}{4}} \hat{V}_{c} \bar{t}+\hat{\kappa}^{\frac{3}{4}} \bar{Y}_{1}+\ldots
\end{aligned}
$$

In contrast to the initial planing phase during which the trailing edge evolves on a much larger time scale when compared with that of the leading edge, in the second phase the body's trailing edge moves on a scale comparable to that of 


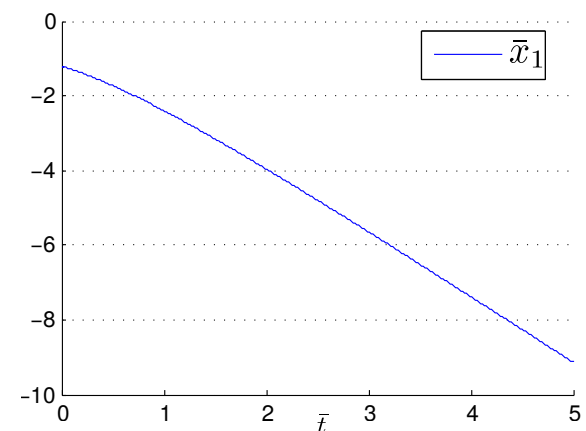

(a) $\bar{x}_{1}$

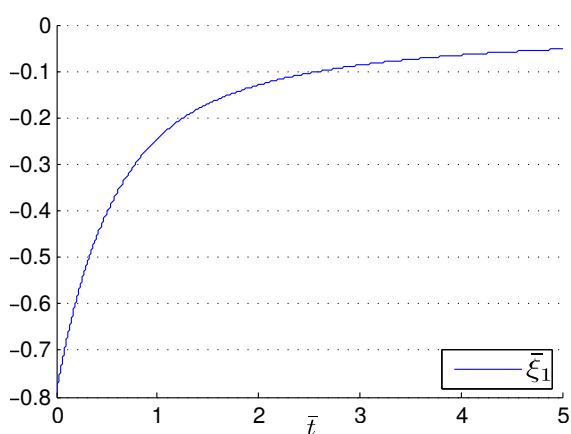

(b) $\bar{\xi}_{1}$

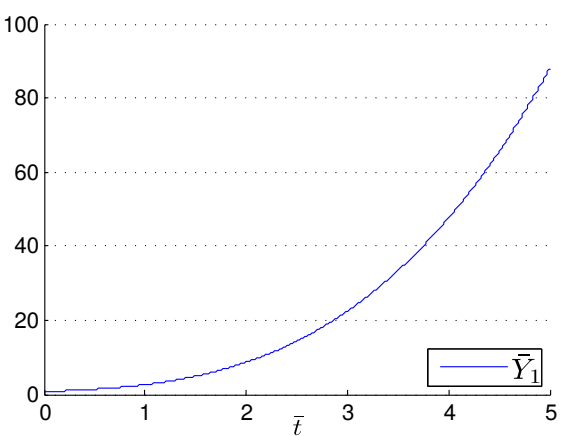

(c) $\bar{Y}_{1}$.

Fig. 10. Plots of $\bar{x}_{1}, \bar{\xi}_{1}$ and $\bar{Y}_{1}$ over time $\bar{t} \in[0,5]$. The values of $\hat{x}_{c 1}, \hat{x}_{c 2}, \hat{Y}_{c}$ and $\hat{V}_{c}$ are the results from the final stage of impact model (18), their respective values are: $-1.9944,0.9434,-0.4431$ and 0.0359 .

the leading edge with only an $O\left(\hat{\kappa}^{\frac{3}{4}}\right)$ adjustment. Substituting these asymptotic expansions into the planing system of (20) yields:

$$
\begin{array}{ll}
\bar{\xi}_{1} \ddot{\bar{Y}}_{1}+1=0, & (32 \mathrm{a}) \\
\frac{1}{2}(6 M)^{\frac{2}{3}} \ddot{\bar{Y}}_{1}-\left[\left(2 \hat{x}_{c 1}+\hat{\omega}_{0} \hat{t}_{N}\right) \bar{x}_{1}+\left(\hat{\omega}_{0} \hat{x}_{c 1}+\hat{V}_{c}\right) \bar{t}\right] \dot{\bar{x}}_{1}^{2}=0, & (32 \mathrm{~b}) \\
{\left[\left(2 \hat{x}_{c 1}+\hat{\omega}_{0} \hat{t}_{N}\right) \bar{x}_{1}+\left(\hat{\omega}_{0} \hat{x}_{c 1}+\hat{V}_{c}\right) \bar{t}\right] \ddot{\bar{x}}_{1}+\left(2 \hat{x}_{c 1}+\hat{\omega}_{0} \hat{t}_{N}\right) \dot{\bar{x}}_{1}^{2}+2\left(\hat{\omega}_{0} \hat{x}_{c 1}+\hat{V}_{c}\right) \dot{\bar{x}}_{1}=0 .}
\end{array}
$$

This system can be solved explicitly via the method of matched asymptotic expansions (see [9]) and the solutions are:

$$
\begin{aligned}
& \bar{x}_{1}=\frac{\left(\gamma_{0}^{2} \bar{t}^{2}-2 \lambda_{0} \Phi_{0} \zeta_{0}\right)^{\frac{1}{2}}-\gamma_{0} t}{\lambda_{0}} \\
& \bar{Y}_{1}=(6 \hat{M})^{-\frac{2}{3}}\left[\frac{2}{3} \frac{\left(\gamma_{0}^{2} \bar{t}^{2}-2 \Phi_{0} \zeta_{0} \lambda_{0}\right)^{\frac{3}{2}}-\gamma_{0}^{3} \bar{t}^{3}}{\lambda_{0}^{2}}+\frac{2 \Phi_{0} \zeta_{0} \gamma_{0} \bar{t}}{\lambda_{0}}\right] \\
& \bar{\xi}_{1}=-\frac{(6 \hat{M})^{\frac{2}{3}} \lambda_{0}^{2}\left(\gamma_{0}^{2} \bar{t}^{2}-2 \Phi_{0} \zeta_{0} \lambda_{0}\right)^{\frac{1}{2}}}{2 \gamma_{0}^{2}\left[\left(\gamma_{0}^{2} \bar{t}^{2}-2 \Phi_{0} \zeta_{0} \lambda_{0}\right)^{\frac{1}{2}}-\gamma_{0} \bar{t}\right]^{2}} .
\end{aligned}
$$

Here $\gamma_{0}=\hat{\omega}_{0} \hat{x}_{c 1}+\hat{V}_{c}$ and $\lambda_{0}=2 \hat{x}_{c 1}+\hat{\omega}_{0} \hat{t}_{N}$. See figure 10 for a demonstration of the solutions.

The solutions indicate that the leading and trailing edge positions in this phase continue to move in the upstream direction. The pace at which the body emerges from the water significantly increases. It becomes evident that as time progresses our planing system will emerge from the $O\left(\hat{\kappa}^{\frac{1}{4}}\right)$ regime, which leads to the final large time phase of this planing. 


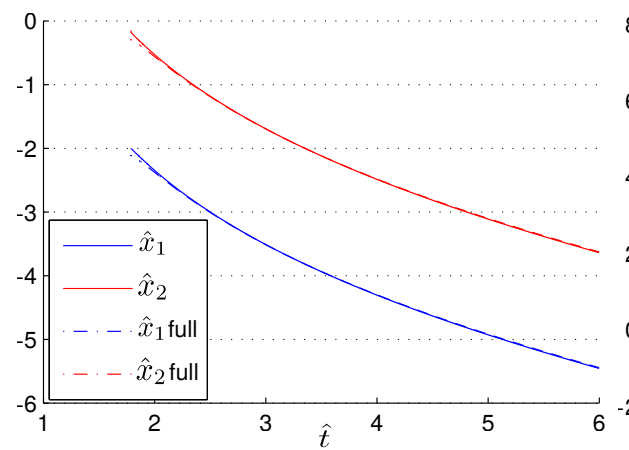

(a)

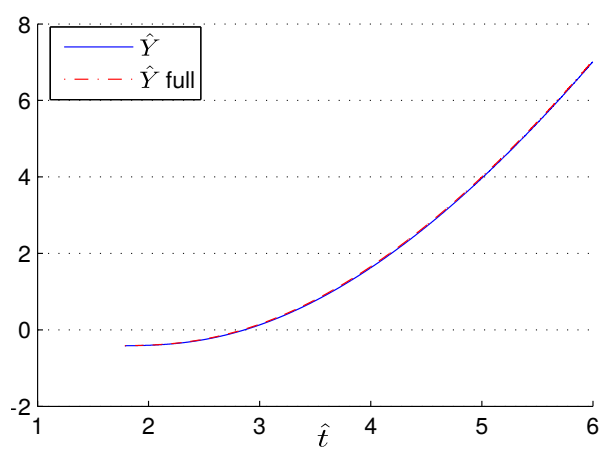

(b)

Fig. 11. Numerical solutions of the planing system (35) compared with the full planing system (20) for $\hat{t}>\hat{t}_{N}$ and $\hat{\kappa}=10^{-4}$. (a) Comparison of numerical solutions for $\hat{x}_{1}$, $\hat{x}_{2}$. (b) Comparison of numerical solutions for $\hat{Y}$.

Planing phase III. In the final phase of the planing motion the system variables can be expanded asymptotically as

$$
\begin{aligned}
& \hat{t}=\breve{t}, \\
& \hat{x}_{1}=\breve{x}_{1}, \\
& \hat{\xi}_{1}=(6 M)^{\frac{1}{3}}-\hat{\kappa} \breve{\xi}_{1}, \\
& \hat{Y}_{1}=\breve{Y}_{1} .
\end{aligned}
$$

Substituting these expansions into the planing system of (20) yields the following planing system for phase III:

$$
\begin{aligned}
& \breve{\xi}_{1} \ddot{\breve{Y}}_{1}+1=0 \\
& \frac{1}{2}(6 M)^{\frac{2}{3}} \ddot{Y}_{1}-\left(\breve{x}_{1}^{2}+\hat{\omega}_{0} \breve{t}_{1}+\breve{Y}_{1}\right)\left(\dot{\breve{x}}_{1}\right)^{2}=0,
\end{aligned}
$$

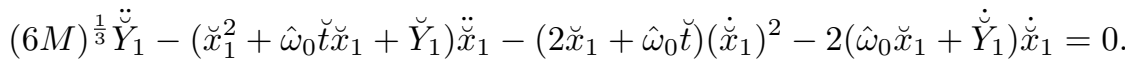

It is difficult to obtain explicit solutions of this coupled non-linear ODE system; numerical solutions are therefore pursued and the results are presented in figure 11. We also present a comparison with the numerical solutions to the full planing system (20). The results show that our phase III planing system is able to capture the planing body's behaviour at large times well.

The solutions indicate that during this phase the planing body continues to emerge from the water and leading and trailing edges continue to move in the upstream direction, with the distance between the two edges fixed at $(6 M)^{\frac{1}{3}}$ at leading order. 
In order to see the behaviour of the system at large times, i.e. as $\breve{t} \rightarrow \infty$, we introduce the following scaled large time variables:

$$
\tilde{t}=\delta \breve{t}, \quad \tilde{x}_{1}=\delta \breve{x}_{1}, \quad \tilde{\xi}=\breve{\xi}, \quad \tilde{Y}=\delta^{2} \breve{Y},
$$

where $\delta \ll 1$ and the tilde sign is used to denote variables which are $O(1)$ at large times. Upon substituting the above variables into the phase III planing system (35) we obtain the system:

$$
\begin{aligned}
& \tilde{\xi}_{1} \ddot{\tilde{Y}}_{1}+1=0, \\
& \left(\tilde{x}_{1}^{2}+\hat{\omega}_{0} \tilde{t} \tilde{x}_{1}+\tilde{Y}\right) \dot{\tilde{x}}_{1}^{2}=0, \\
& \left(\tilde{x}_{1}^{2}+\hat{\omega}_{0} \tilde{t} \tilde{x}_{1}+\tilde{Y}\right) \ddot{\tilde{x}}_{1}+\left(2 \tilde{x}_{1}+\hat{\omega}_{0} \tilde{t}\right) \dot{\tilde{x}}_{1}^{2}+2\left(\hat{\omega}_{0} \tilde{x}_{1}+\dot{\tilde{Y}}\right) \dot{\tilde{x}}_{1}=0 .
\end{aligned}
$$

The solutions here can be written down explicitly with the initial conditions of $\tilde{x}_{1}(0)=0$ and $\tilde{Y}(0)=0$ :

$$
\begin{aligned}
& \tilde{x}_{1}=-\frac{1}{2} \hat{\omega}_{0} \tilde{t}, \\
& \tilde{\xi}=-\frac{2}{\hat{\omega}_{0}^{2}}, \\
& \tilde{Y}=\frac{1}{4} \hat{\omega}_{0}^{2} \tilde{t}^{2} .
\end{aligned}
$$

If we could see the planing body lifting off and separating from water, the positions of the leading and trailing edges should eventually coincide as shown by [42]. This is however not the case in our linearised planing model as the large time solutions (37) demonstrate: under our linearised planing regime the body continues to lift upwards as time grows but the distance between the two wetted edges does not decrease. As the body evolves vertically to the squared power of time it will eventually depart from the linearised regime defined in (17), as which point we will need to revert to the full planing system to capture the motion of the planing body.

\section{Post-liftoff and fly-away of a body from a surface}

In this section, post-liftoff of a thin body and its continued departure from the ground (fly-away) are investigated. We model, analyse and compute the mechanisms for a single body which is initially at rest on a horizontal solid surface and can be lifted off impulsively by a horizontal fluid flow. The aim is to gain an understanding of lift-off followed by a return to the surface or complete fly-away. Criteria for liftoff and fly-away and the ensuing motion are examined. Analysis of the early behaviour when lift-off starts and a numerical study of the ensuing evolution are presented, followed by large-time analysis which shows a critical flow speed for fly-away for any shape of body.

The body is assumed much denser than the fluid, implying that the major gravity force acts on the body. Experimental results or observations are many, 


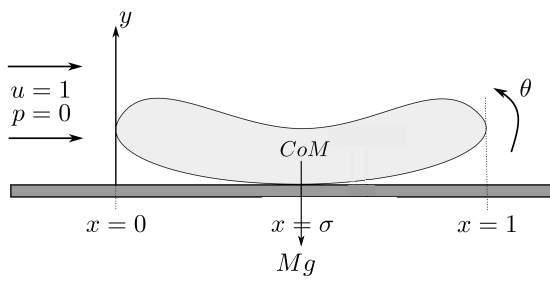

(a) At time $t=0$.

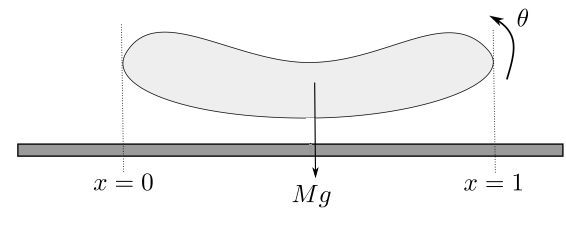

(b) At time $t>0$.

Fig. 12. (a) A sketch of the body at its initial position, the fixed centre of mass (CoM), the contact point $x=\sigma$ at time $t=0$ and the oncoming stream of fluid. (b) The body position at some time $t>0$.

as in [13], [18], [19]. Applications vary from grain segregation, leaf-blowers, dust loss, dust blowing, removal of debris, sand movement on beaches, to aircraft take-off and ski jumping. See for example [15], [16], [22].

The fluid is incompressible and its motion is assumed to be two-dimensional and laminar with uniform density $\rho^{*}$, where the asterisk $\left(^{*}\right)$ refers to a dimensional quantity. We express the motion of the fluid and the immersed thin body (see figure 12) with regard to non-dimensional flow velocities $(u, v)$, corresponding Cartesian coordinates (horizontal $x$, vertical $y$ ), time $t$ and pressure $p$, such that the dimensional versions are $u^{*}(u, v), L_{1}^{*}(x, y), L_{1}^{*} t / u^{*}$ and $\rho^{*} u^{* 2} p$, in turn. Here $L_{1}^{*}$ is the length of the body, while $u^{*}$ is the free-stream velocity and the temporal factor $L_{1}^{*} / u^{*}$ is the typical transport time. The Reynolds number Re is large. Also $(u, v)$ is given by $(1,0)$ in the far field and the leading edge of the body can be taken as origin.

A nonlinear evolutionary system for the unknown scaled functions $h, \theta, u, p$ is found to govern the interaction. Here $x=\sigma$ is the prescribed $x$-location of the centre of mass and the initial contact point with the ground. Also $h(t)$ is the vertical $y$-location of the centre of mass of the body, while $\theta(t)$ is the small angle the body chord makes with the horizontal.

First, we present the model in detail, followed by the behaviour for small times. Then we examine the lift-off criterion for different body shapes. If the body does lift off it returns to the ground within a finite time or flies away at large time. We focus on the latter, finding a criterion for fly-away. Finally, we provide conclusions and further discussion.

\subsection{Governing equations and the parameters}

The interaction between fluid and body and governing equations are described as follows. The body has a smooth shape and is thin, of vertical scale $O(\delta)$ 
with $\delta$ being small. The fixed surface or wall is located at $y=0$ on which the body initially is at rest and in contact, directly below its centre of mass whose $x$-location is $x=\sigma$ as presented in figure 12a. The incoming flow moving from left to right is the uniform stream with $(u, v)=(1,0)$.

The scaled body mass and the scaled acceleration due to gravity are represented as $M$ and $g$ respectively in figure 12. Also $I$ denotes the moment of inertia given below. The scaled weight of the body is represented as $M g$. The flow over the length scale of order unity remains irrotational to leading order almost everywhere and the scaled vorticity is zero under the present assumptions. The velocity $u=u(x, t)$ does not depend on $y$, a feature which is due to the thin layer dynamics. Then $v$ is forced through continuity to change in $y$ from zero at zero $y$ to a value consistent with the kinematic condition at the unknown position of the moving lower surface of the body.

The equations of motion of the body are as in $(5 \mathrm{~b}, 5 \mathrm{c})$ such that

$$
\begin{aligned}
& M\left(\frac{d U_{B}}{d t}+g\right)=\int_{0}^{L} p d x, \\
& \frac{d\left(I \Omega_{B}\right)}{d t}=\int_{0}^{L}(x-\sigma) p d x,
\end{aligned}
$$

where $L$ is the length of the body, which is taken as unity in this section. Also $U_{B}=\dot{h}$ is the translational velocity and $\Omega_{B}=\dot{\theta}$ is the angular velocity. The dimensional mass is $\rho^{*} L_{1}^{*} M \delta^{-1}$, while the dimensional moment of inertia is $\rho^{*} L_{1}^{*}{ }^{4} I \delta^{-1}$. The acceleration due to gravity is $\delta u^{* 2} g L_{1}^{*-1}$ in dimensional terms. The Froude number is $(\delta g)^{-1}$, whereas the Richardson number is $\delta g$. Here $I<$ $M / 4$ from its definition.

The equations of motion within the fluid gap are shallow-water equations as in $(4 \mathrm{a}, 4 \mathrm{~b})$,

$$
\begin{aligned}
& H_{t}+(u H)_{x}=0, \\
& u_{t}+u u_{x}=-p_{x} .
\end{aligned}
$$

Here $H(x, t)$ denotes the unknown scaled thickness of the thin gap and depends on the lower surface shape of the body and its orientation given in (39c) below; $F^{-}(x)$ is the prescribed shape of the underbody as in section 2 . The kinematic condition yields (39a) while the dominant streamwise momentum balance is given as (39b). Here $p$ is dependent only on $x, t$ by virtue of the normal momentum balance. Changes in the lateral location and orientation of the body lead to the contributions $h$ and $\theta$ respectively. These are prescribed at the initial time of zero as in $(39 \mathrm{~d})$. Thus

$$
\begin{gathered}
H(x, t)=-F^{-}(x)+h(t)+(x-\sigma) \theta(t), \\
h(0)=F^{-}(\sigma) ; \quad \theta(0)=F^{-'}(\sigma) .
\end{gathered}
$$

We also have the Euler jump across the leading-edge Euler zone at $x=0+$ such that

$$
p+\frac{1}{2} u^{2}=\frac{1}{2} \quad \text { at } \quad x=0+
$$


and the Kutta condition at the trailing edge of the body at $x=1$ yields

$$
p=0 \quad \text { at } \quad x=1 .
$$

The constraints $(39 \mathrm{~d})$ coming from $H=0$ at the contact point $x=\sigma$, and also $\partial H / \partial x=0, x=\sigma$, hold for the smooth shapes considered herein. The leadingedge jumps (39e) are required in order to satisfy the equi-pressure condition at the trailing edge (see section 2.1). These conditions are coupled with the bodymotion equations in $(38 \mathrm{a}, \mathrm{b})$. The task in general is to solve the nonlinear system (38a,b) and (39a-f) for the unknowns $h, \theta, u, p$.

\subsection{Small-time behaviour}

When the fluid flow is begun impulsively or a considerable change in the fluid flow occurs at time $t=0$, the body is supposed to be positioned initially on the surface. Then it is assumed to move from rest. For $0<t \ll 1$, two regions are present: one is influenced by the whole underbody for $0<x<\sigma, \sigma<x<1$ and the other is in the neighbourhood of the contact point $\sigma$. The task now is to study the mathematical structure of the fluid-body interaction as the lifting begins and the contributions of the inner and outer region dynamics.

In the outer region, the appropriate expansions for height and orientation have the patterns above with the typical $x \sim O(1)$. The small-time perturbations of the vertical location and the orientation of the centre of mass are predicted as

$$
(h, \theta)=\left(h_{0}, \theta_{0}\right)+t\left(h_{1}, \theta_{1}\right)+\ldots,
$$

where the dominant terms $\left(h_{0}, \theta_{0}\right)=\left(F^{-}(\sigma), F^{-^{\prime}}(\sigma)\right)$ are found from $(39 \mathrm{~d})$ and the contact point $x=\sigma$ is also the horizontal location of the centre of mass. Hence the gap width, the scaled velocity and pressure $H, u, p$ develop according to

$$
(H, u, p)=\left(H_{0}(x), u_{0}(x), p_{0}(x)\right)+t\left(H_{1}(x), u_{1}(x), p_{1}(x)\right)+\ldots,
$$

with the leading order term being $H_{0}(x)=-F^{-}(x)+h_{0}+(x-\sigma) \theta_{0}$ where $h_{0}$ and $\theta_{0}$ are given in (40a). The unknown perturbations $u_{0}, u_{1}, p_{0}, p_{1}$ are functions of $x$ to be determined. Substituting (40b) into (39a) and integrating in $x$ leads to the leading order term of the velocity in the outer region,

$$
u_{0}(x)=\frac{1}{H_{0}(x)}\left\{-h_{1}(x-\sigma)-\frac{\theta_{1}}{2}(x-\sigma)^{2}+c_{1}\right\},
$$

where $c_{1}$ is the integration constant. A requirement for definiteness of $u_{0}$ at the contact position $x=\sigma$ is applied in order to keep the related pressure coefficient finite at the initial contact location in accordance with the fluid-body interaction structure. Indeed, this indicates that $c_{1}=0, h_{1}=0$ to keep $u_{0}$ finite 
at $x=\sigma$ since the denominator $H_{0}$ is then of $O\left((x-\sigma)^{2}\right)$ there. Making another substitution into (39a) with (41a) and (40b) and an integration in $x$ yields

$$
u_{1}(x)=\frac{1}{H_{0}(x)}\left\{-2 h_{2}(x-\sigma)-\frac{\theta_{2}}{2}(x-\sigma)^{2}-c_{2}\right\}+\frac{1}{H_{0}(x)^{2}}\left\{\frac{\theta_{1}^{2}}{2}(x-\sigma)^{3}\right\} .
$$

Next, integrating (39b) with respect to $x$ at leading order and considering $(39 \mathrm{e}, \mathrm{f})$ yields the leading term in the pressure as

$$
p_{0}(x)= \begin{cases}-\int_{0}^{x} u_{1}(\hat{x}) d \hat{x}-\frac{1}{2} u_{0}(x)^{2}+\frac{1}{2}, & x \in[0, \sigma), \\ -\int_{1}^{x} u_{1}(\hat{x}) d \hat{x}-\frac{1}{2}\left\{u_{0}(x)^{2}-u_{0}(1)^{2}\right\}, & x \in(\sigma, 1] .\end{cases}
$$

Matching below implies that $c_{2}$ in (41b) is zero. This reduces the local inertial effects. We will study the case $\theta_{1}=0$ implying $u_{0}=0$ in the rest of the section. (See also [10] for the case $\theta_{1} \neq 0$ whereby $u_{0} \neq 0$ ). We also observe that

$$
\begin{gathered}
\qquad p_{0} \sim \frac{2 h_{2}}{\mathcal{B}} \ln |x-\sigma|+\pi_{ \pm} \quad \text { as } \quad x \rightarrow \sigma_{ \pm}, \\
\text {where } \quad \pi_{+}=-\int_{1}^{\sigma} u_{1} d x, \quad \pi_{-}=\frac{1}{2}-\int_{0}^{\sigma} u_{1} d x
\end{gathered}
$$

and $\mathcal{B}=F^{-\prime \prime} / 2$ from the expression for the gap width near the original contact point. Figure 13 shows a sample comparison between $p_{0}$ in (43a) and a numerical solution for the pressure given in [10]. The inner region near the initial contact $x=\sigma$ is investigated next.

For $x$ near the lift-off point, $x=\sigma+t \eta$ with $\eta \sim O(1)$, the solution takes the form

$$
(H, u, p)=\left(t^{2} H_{2}^{*}(\eta), u_{0}^{*}(\eta), \frac{2 h_{2}}{\mathcal{B}} \ln (t)+p_{0}^{*}(\eta)\right)+\ldots .
$$

These scalings stem from those in the outer region. Substituting into (39a-d) and matching yields the leading order terms in the local velocity and pressure expansion such that

$$
\begin{gathered}
u_{0}^{*}(\eta)=\frac{-2 \eta h_{2}-c_{0}^{*}}{\mathcal{B} \eta^{2}+h_{2}}, \\
p_{0}^{*}(\eta)=p_{0}^{*}(0)+\eta u_{0}^{*}(\eta)-\int_{0}^{\eta} u_{0}^{*} d \eta-\frac{1}{2} u_{0}^{*}(\eta)^{2}+\frac{1}{2} u_{0}^{* 2}(0),
\end{gathered}
$$

where $c_{0}^{*}, p_{0}^{*}(0)$ are integration constants to be determined. Matching the velocities and pressures in the inner and outer regions yields $c_{2}=0$ in (41b) for $u_{0}=0$ in (41a). Also $c_{0}^{*}$ is determined by

$$
\frac{\sqrt{2} \pi c_{0}^{*}}{\left(h_{2} F^{-\prime \prime}\right)^{1 / 2}}=-\int_{1}^{\sigma} u_{1} d x-\frac{1}{2}+\int_{0}^{\sigma} u_{1} d x .
$$

The pressure coefficient $p_{0}^{*}(0)$ can similarly be found, and this completes the velocity and pressure solutions. 


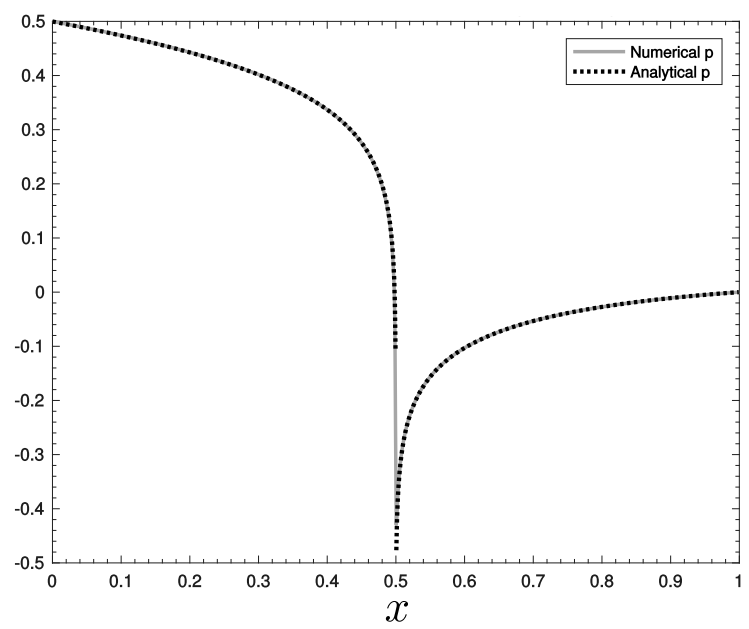

Fig. 13. Comparison between analytical and numerical solutions of the pressure at time $t$ almost zero.

We note that the main body motion is only controlled by the outer region. The body-movement relations $(38 \mathrm{a}, \mathrm{b})$ give the leading order contributions

$$
\begin{gathered}
M\left(2 h_{2}+g\right)=\int_{0}^{\sigma} p_{0}(x, t) d x+\int_{\sigma}^{1} p_{0}(x, t) d x \\
2 I \theta_{2}=\int_{0}^{\sigma}(x-\sigma) p_{0}(x, t) d x+\int_{\sigma}^{1}(x-\sigma) p_{0}(x, t) d x .
\end{gathered}
$$

We need $h_{2}$ in (48a) to be positive so that the body can lift off from the surface. Substituting $p_{0}(x)$ from (42) (for $u_{0}=0$ ) into the system (48a,b) gives two linear equations for the two unknowns $h_{2}, \theta_{2}$, namely

$$
\begin{gathered}
2 M h_{2}=\frac{\sigma}{2}+h_{2} \mathcal{J}_{1}+\theta_{2} \mathcal{J}_{2}-W, \\
2 I \theta_{2}=\frac{-\sigma^{2}}{4}+h_{2} \mathcal{J}_{2}+\theta_{2} \mathcal{J}_{3},
\end{gathered}
$$

with

$$
\mathcal{J}_{1}=-2 \int_{0}^{1} \frac{(x-\sigma)^{2}}{H_{0}(x)} d x, \quad \mathcal{J}_{2}=-\int_{0}^{1} \frac{(x-\sigma)^{3}}{H_{0}(x)} d x, \mathcal{J}_{3}=-\frac{1}{2} \int_{0}^{1} \frac{(x-\sigma)^{4}}{H_{0}(x)} d x
$$

The coefficient $\mathfrak{J}_{2}$ is identically zero if the body is symmetric with $\sigma=1 / 2$. 


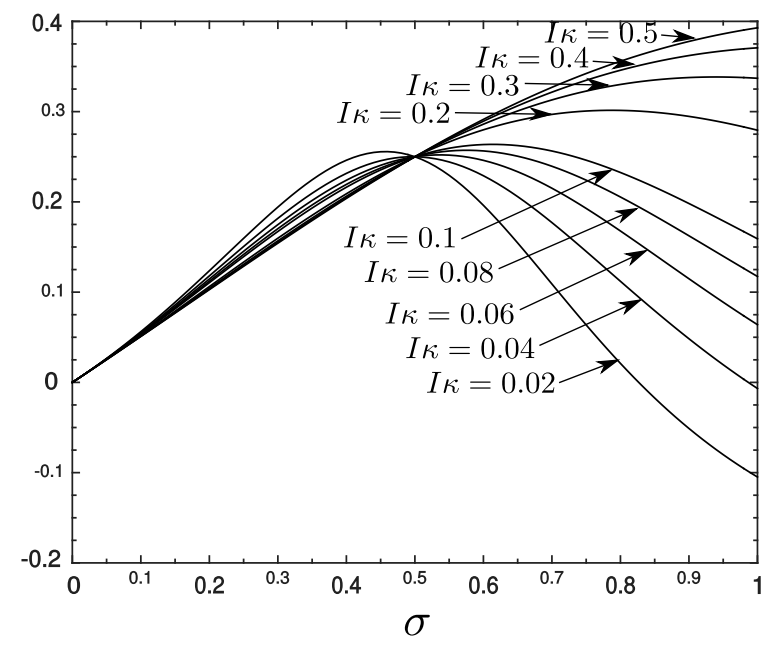

Fig. 14. The right hand side of (51) versus $\sigma$ for varying $I \kappa$ ranging from 0.02 to 0.5 . The underbody has a parabolic shape.

\subsection{Lift-off criterion}

The study suggests that the lift-off requirement is simply $h_{2}>0$. Considering a body having general shape and using the relationship $\theta_{2}=\left(h_{2} J_{2}-\sigma^{2} / 4\right) / \beta$ in (49b) where $\beta=\left(2 I-\mathcal{J}_{3}\right)$, (49a) becomes

$$
\begin{gathered}
\gamma h_{2}=\frac{\sigma}{2}-W-\frac{\mathcal{J}_{2}}{\beta} \frac{\sigma^{2}}{4}, \\
\text { with } \gamma=\left(\alpha-\frac{\mathcal{J}_{2}^{2}}{\beta}\right), \alpha=\left(2 M-\mathcal{J}_{1}\right) .
\end{gathered}
$$

We note that $\mathfrak{J}_{2}$ can either be negative or positive, while $\alpha>0, \beta>0, J_{1}<$ $0, \mathcal{J}_{3}<0$. If $M$ and $I$ are sufficiently large that $\gamma>0$, then from (50a) lift-off requires $M g<\frac{\sigma}{2}-\frac{\sigma^{2}}{4 \beta} \mathrm{J}_{2}$ by virtue of $h_{2}>0$. The criterion in (50a) becomes

$$
M g<\frac{\sigma}{2}+\frac{\sigma^{2}}{8}(1-2 \sigma)\left(2 I \kappa+\frac{1}{6}(1-\sigma)^{3}+\sigma^{3}\right)^{-1}
$$

for a parabolic shaped body $F^{-}(x)=\kappa x(1-x)$ and for any $\sigma$.

The right hand side of (51) versus $\sigma$ is shown in figure 14 for a range of $I \kappa$ values. The value of $M g$ needs to be small for lift-off at small $\sigma$ values. When $\sigma$ is near unity, lift-off is possible for larger $M g$. When the position $\sigma$ is away from the leading and trailing edges, lift-off does not occur for a significant range of $\sigma$. The lift-off requirement is $M g<1 / 4$ at $\sigma=1 / 2$, in accordance with (49a).

Numerical evolutions of the system (38a-39f) are shown in figures 15,16 . The body lifts off but returns to the ground after a finite time in figure 15, whereas in 


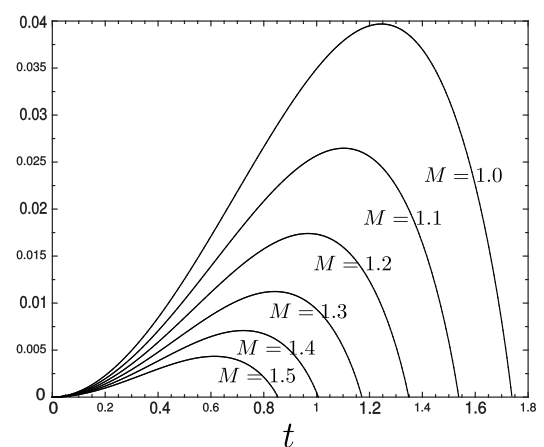

(a)

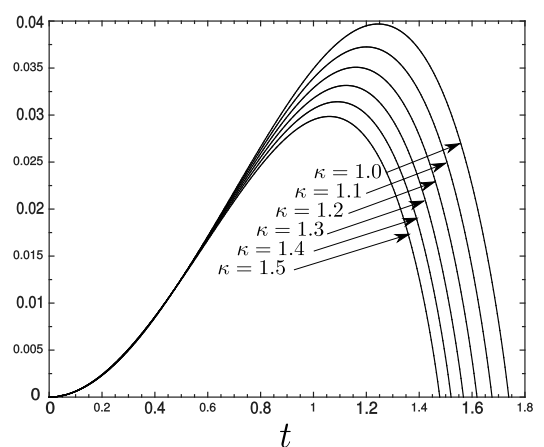

(b)

Fig. 15. Numerical solutions of $H_{\min }$ against $t$ in which the body returns to the surface. The effect of (a) scaled mass $M$ ranging from 1 to 1.5 , (b) $\kappa$ ranging from 1 to 1.5 . In (a), the scaled moment of inertia $I$ also changes according to $I=\Gamma M$, for $\Gamma=0.2$. Here a symmetrical sinusoidal shaped body is investigated: $F^{-}=\kappa \sin (\pi x)$.

figure 16 the departure from the ground continues to large times and produces a fly-away phenomenon with $h, \theta$ becoming large then.

On the other hand, we might expect the time scales of $(38 \mathrm{a}, \mathrm{b})$ to dominate for large $M, I$. Therefore, the time scale is given as $t=M^{1 / 2} T$ with $T$ being of order unity since the typical $h, p, \theta$ must remain of $O(1)$. We also take $I=M \Gamma$ with $\Gamma$ being $O(1)$ and $g \sim O\left(M^{-1}\right)$, keeping weight $W$ of order unity. Solving $(39 a, b)$ with the new scalings gives more explicit velocity and pressure solutions and leads to

$$
\begin{gathered}
\ddot{h}=\frac{1}{2} \int_{0}^{1}\left(1-(h(T)+(1-\sigma) \theta(T))^{2} H(x, T)^{-2}\right) d x-W, \\
\Gamma \ddot{\theta}=\frac{1}{2} \int_{0}^{1}(x-\sigma)\left(1-(h(T)+(1-\sigma) \theta(T))^{2} H(x, T)^{-2}\right) d x .
\end{gathered}
$$

Subsequent working shows that two coupled ordinary differential equations are obtained for $h, \theta$ for the parabolic underbody. Furthermore, in the general case a large-time response is suggested by $(52 \mathrm{a}, \mathrm{b})$ in which $h, \theta$ grow like $t^{2}$ leading to the integrands being of order unity and an independence from the shape $F^{-}(x)$. This is followed through below in the general case.

\subsection{Large-time behaviour}

At large times a first guess for the response is that $h, \theta$ are of $O\left(t^{2}\right)$ as $p$ typically must be $O(1)$ by virtue of $(39 \mathrm{e})$. The analysis here is for $t \gg 1$ and general values of $M, I, g$. We therefore seek an asymptotic description taking the form

$$
(H, h, \theta, u, p)=\left(t^{2} H_{2}(x), t^{2} h_{2}, t^{2} \theta_{2}, u_{0}(x), p_{0}(x)\right)+\ldots
$$




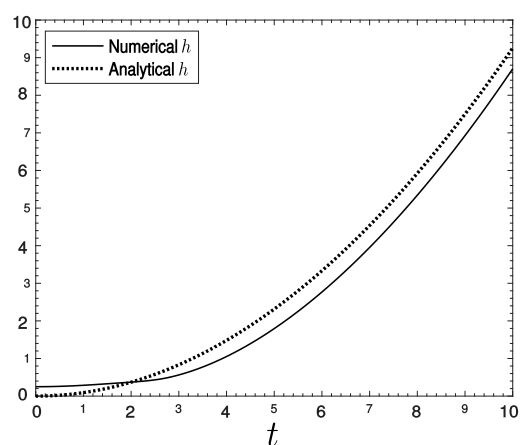

(a)

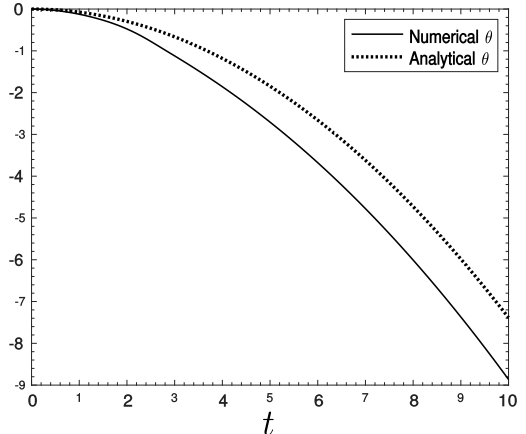

(b)

Fig. 16. (a) Comparison between analytical and numerical solutions for (a) $h$, (b) $\theta$ for a fly-away case. Body has symmetric parabolic shape $F^{-}=x(1-x), \sigma=0.5$, scaled mass $M=1$, moment of inertia $I=0.2$, and $g=0.1$.

Simple quasi-steady relations hold again as the time derivatives are negligible in $(39 a, b)$. Likewise, the body-shape contribution $F^{-}(x)$ becomes negligible in comparison with the $h, \theta$ contributions. The Kutta condition (39f) implies $u_{0}(1)=1$. Thus, the leading-order terms in the velocity and the pressure $u_{0}, p_{0}$ are found as

$$
\begin{gathered}
u_{0}(x)=\frac{h_{2}+(1-\sigma) \theta_{2}}{h_{2}+(x-\sigma) \theta_{2}}, \\
p_{0}(x)=\frac{1}{2}\left\{1-\left\{\frac{h_{2}+(1-\sigma) \theta_{2}}{h_{2}+(x-\sigma) \theta_{2}}\right\}^{2}\right\} .
\end{gathered}
$$

The pressure in (54b) is found by substituting (54a) into (39e). Finally, the body-balance equations in $(38 \mathrm{a}, \mathrm{b})$ yield

$$
\begin{gathered}
2 M h_{2}=-\frac{\theta_{2}}{2\left(h_{2}-\sigma \theta_{2}\right)}-W \\
4 \Gamma M \theta_{2}=\left(\frac{1}{2}-\sigma\right)+\frac{h_{2}}{\theta_{2}}\left(\frac{h_{2}+(1-\sigma) \theta_{2}}{h_{2}-\sigma \theta_{2}}\right)-\frac{1}{\theta_{2}^{2}}\left(h_{2}+(1-\sigma) \theta_{2}\right)^{2} \ln \left|\frac{h_{2}+(1-\sigma) \theta_{2}}{h_{2}-\sigma \theta_{2}}\right| .
\end{gathered}
$$

The $t^{2}$ terms $h_{2}, \theta_{2}$ are thus determined, following which the leading order terms $H_{2}, u_{0}, p_{0}$ in (53) can then be obtained.

A sample comparison between the analytical solutions and numerical ([10]) for the height $h$ and angle $\theta$ is shown in figure 16. The analytical solutions use the large-time asymptotic expansions in $(55 \mathrm{a}, \mathrm{b})$. There is a close match for both $h, \theta$ in terms of the growth with time.

Figure 17 plots the coefficients $h_{2}, \theta_{2}$ versus scaled weight. A critical value $W=W_{c}(=1 / 2)$ emerges for the fly-away. To explain this we put

$$
M g=\frac{1}{2}-\varepsilon \quad \text { with } \varepsilon \ll 1
$$




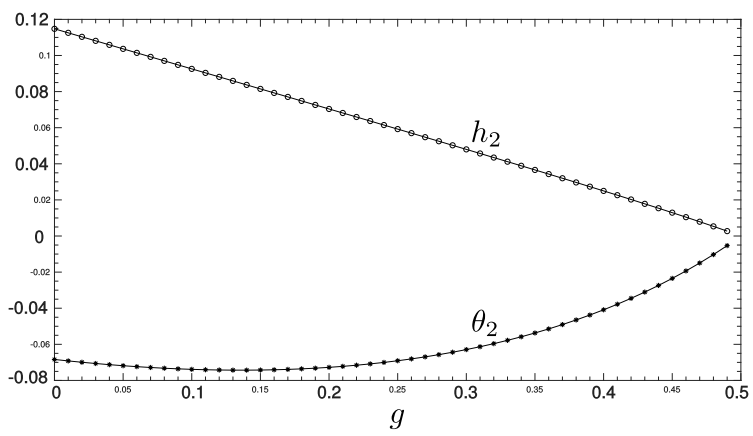

Fig. 17. The coefficients $h_{2}, \theta_{2}$ in large time response versus weight: $0<M g<0.5$ for $\sigma=0.5, M=1$. This indicates the range for lift-off and fly-away for any body shape: see text.

for some positive $\varepsilon$ and then expand $h_{2}, \theta_{2}$ in powers of $\varepsilon$ such that $\left(h_{2}, \theta_{2}\right)=$ $\varepsilon\left(\hat{h}_{2}, \hat{\theta}_{2}\right)+\ldots$ It is found with the scaled mass $M=1$ for example that

$$
\left(h_{2}, \theta_{2}\right)=\left(\frac{\varepsilon}{2},-\varepsilon\right)+\ldots
$$

The critical value $1 / 2$ applies for any shape of body. As $h_{2}, \theta_{2}$ are small, the Bernoulli pressure head drives the main balance of forces which is between the weight and the pressure force in the critical case. The asymptotic predictions in (57) show a close agreement with those in figure 17 as $g$ increases. We note that the $\left(h_{2}+(1-\sigma) \theta_{2}\right)$ contribution in $(55 \mathrm{~b})$ implies that the trailing edge stops rising, whereas the leading edge height is still increasing for a symmetric body for example.

\subsection{Summary}

In the present section, liftoff and fly-away of a body from a surface have been investigated. For any symmetric body the critical value of scaled weight $W$ is found to be $1 / 4$ so that the body can initially lift off from the surface. A criterion is also found for nonsymmetric body shapes. After lifting off, the body can either return to the surface or fly away. There is a wide range of parameters such as gravity, scaled mass, moment of inertia as well as body shapes where liftoff can occur in practice. Also, a critical value of the scaled weight is found to be $1 / 2$ for a fly-away and this applies irrespective of the shape of body. Bernoulli pressure during liftoff acts only on the front half of the underbody in contrast with the complete underbody at late times due to the body being far from the wall. The difference between these two factors stems from this fact.

The liftoff and fly-away criteria on the incident velocity can be written in dimensional terms, respectively,

$$
u^{* 2} /\left(h_{B}^{*} g^{*}\right)>4\left(\rho_{B}^{*} / \rho^{*}\right),
$$




$$
u^{* 2} /\left(h_{B}^{*} g^{*}\right)>2\left(\rho_{B}^{*} / \rho^{*}\right),
$$

(for a symmetric body in the case of (58a)) with $g^{*}$ denoting gravity. This is for a body mass represented as $\rho_{B}^{*} h_{B}^{*} L_{1}^{*}$ where $\rho_{B}^{*}$ is the body density and $h_{B}^{*}$ is the mean body thickness. These criteria are on effective Froude numbers and are broadly in line with Shield's condition ([11], [17]) in sediment processes; this section shows evolution towards or away from fly-away and determines a precise coefficient (4 or 2) rather than the order of magnitude estimate of Shield. Further agreement with experiments or observations concerns the movement of dust on the surface of Mars as discussed in [4]. The difference between the liftoff and flyaway conditions implies that symmetric bodies for instance which are subject to flow velocities between the two values in $(58 \mathrm{a}, \mathrm{b})$ satisfy the fly-away condition but are unable to lift off, while a body that lifts off may either return to the ground or fly away.

The effects of incident shear in the oncoming flow, three-dimensionality and viscous effects have not been considered yet. The normal pressure gradient comes into play considerably on a larger time scale. Several bodies, clashes, reptation, body flexibility, and investigating the influence of the surface shape might also be of further concern.

\section{Many bodies in slightly viscous fluid}

Allowing for several plates at small angle of attack in unbounded flow forms a basic part of the extension of the research in section 2.4. The classic exact solution for streaming flow past two cylindrical aerofoils is given by [48] and derived succinctly in [49] where it is extended to include freely propagating point vortices and patches of vorticity within the flow field. [50] extended Lagally's result to flow past a finite stack of cylindrical aerofoils with specified circulations about the aerofoils. Here we consider the case of plates aligned at small angles of attack to the flow. The circulation around each plate is determined uniquely by requiring the flow to leave the trailing edge of the plate smoothly: given the angle of attack of each plate the flow field is unique. This allows the forces on the plates to be computed and thus the subsequent motion of the plates if they are free to move (as in section 2.4).

\subsection{A single plate}

Consider the single plate given by $y=0,|x|<1$ in the infinite complex $z$-plane and take the pre-image of this domain in the complex $\zeta$-plane to be the interior of the unit circle $|\zeta|=1$, denoted $\mathcal{C}$ here. Let what will become the leading edge of the plate, at $z=-1$, be mapped to the point $\zeta=1$ on $\mathcal{C}$ and the trailing edge, at $z=1$, be mapped to the point $\zeta=-1$. Then the conformal mapping between the domains is the Joukowski map

$$
z=-(1 / 2)(\zeta+1 / \zeta), \quad \zeta=-z+\sqrt{z^{2}-1},
$$


with the point at infinity in the $z$-plane mapped to the origin in the $\zeta$-plane.

We wish to construct the analytic function $q(z(\zeta))$ giving the velocity field $q=u+\mathrm{i} v$ that has velocity $v=1$ on the plate and vanishes at infinity. Note that it is the analytical function $q$ that is mapped from the $\zeta$ - to the $z$-plane. The corresponding complex velocity potentials do not map to each other with

$$
w(\zeta)=\int q(\zeta) \mathrm{d} \zeta, \quad W(z)=\int q(z) \mathrm{d} z=\int q(\zeta)(\mathrm{d} z / \mathrm{d} \zeta) \mathrm{d} \zeta .
$$

Observe that the streamlines for a dipole in an unbounded domain are circles, i.e. the imaginary part of the complex potential for a dipole is constant on circles. If the velocity field $q$ in the $\zeta$-plane is itself taken to be a dipole centred at the leading edge $\zeta=1$, directed along the $\Im \zeta$ axis, i.e. $q \sim \mathrm{i} /(\zeta-1)$, then $v=\Im q$ will be constant on circles through $\zeta=1$ and, in particular, on the circle $\mathcal{C}$. To construct this velocity field, introduce in the $\zeta$-plane the complex velocity potential for a vortex of strength $\kappa$ at the leading edge and the corresponding complex velocity

$$
w=(\kappa / 2 \pi \mathrm{i}) \log (\zeta-1), \quad q=(\kappa / 2 \pi \mathrm{i})(\zeta-1)^{-1} .
$$

Then $q \rightarrow 0$ as $\zeta \rightarrow \infty$ as required and on $\mathcal{C}$, where $\zeta=\exp (\mathrm{i} \theta)$,

$$
q=(\kappa / 4 \pi)[-\cot (\theta / 2)+\mathrm{i}],
$$

showing that $v$ is constant on $\mathcal{C}$ and $v=1$ there when $\kappa=-4 \pi$. Note that $u$ vanishes at the trailing edge $\theta= \pm \pi$ and is infinite at the leading edge $\theta=0$. This completes the determination of the complex velocity and complex velocity potential in the $\zeta$-plane, giving

$$
w=2 \mathrm{i} \log (\zeta-1), \quad q=2 \mathrm{i} /(\zeta-1) .
$$

The corresponding complex velocity and complex velocity potential in the $z$-plane are

$$
\begin{aligned}
q(z) & =-2 \mathrm{i} /\left(1+z-\sqrt{z^{2}-1}\right)=-\mathrm{i} \sqrt{z-1} / \sqrt{z+1}-\mathrm{i} \\
W(z) & =-\mathrm{i} \int\left(1 / \zeta+1 / \zeta^{2}\right) \mathrm{d} \zeta=\mathrm{i}(-\log \zeta+1 / \zeta) \\
& =-\mathrm{i}\left[z+\sqrt{z^{2}-1}-\log \left(z+\sqrt{z^{2}-1}\right)\right] .
\end{aligned}
$$

Figure 18 gives the streamlines for this flow.

The Blasius theorems give the $x$ and $y$ components of force, $X$ and $Y$, and the moment $M$ about the origin as

$$
X-\mathrm{i} Y=\frac{1}{2} \mathrm{i} \hat{\rho} \oint \hat{q}^{2} \mathrm{~d} z, \quad M=-\frac{1}{2} \hat{\rho} \Re \oint \hat{q}^{2} z \mathrm{~d} z,
$$

where $\hat{\rho}$ is the fluid density, $\hat{q}$ is the total fluid velocity, and the integral is taken around the plate and outside the singularity at the leading edge. Now $\hat{q}=U+q$, 


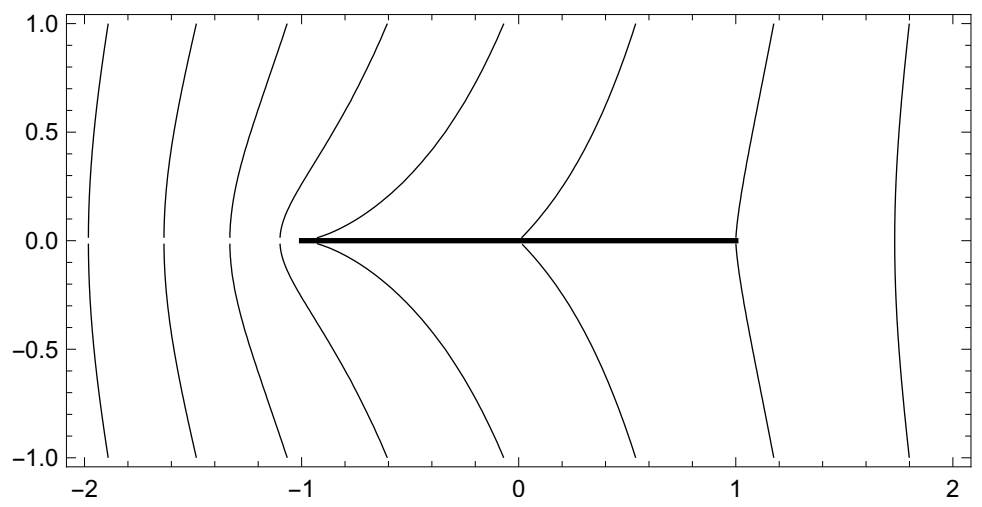

Fig. 18. Perturbation streamlines for potential flow past a single plate at small angle of attack.

where $U$ is the oncoming flow so $\hat{q}^{2}=U^{2}+2 U q+q^{2}$ with the first term being constant and thus making no contribution and the final term negligible in the linear approximation. Hence in the linear approximation

$$
X-\mathrm{i} Y=\mathrm{i} \hat{\rho} U \oint q(z) \mathrm{d} z, \quad M=-\hat{\rho} U \Re \oint q(z) z \mathrm{~d} z .
$$

Since the velocity fields in $\zeta$ and $z$ map to each other, these formulae become immediately

$$
X-\mathrm{i} Y=\mathrm{i} \hat{\rho} U \oint_{\mathcal{C}^{\prime}} q(\zeta)(\mathrm{d} z / \mathrm{d} \zeta) \mathrm{d} \zeta, \quad M=-\hat{\rho} U \Re \oint_{\mathcal{C}^{\prime}} q(\zeta) z(\zeta)(\mathrm{d} z / \mathrm{d} \zeta) \mathrm{d} \zeta
$$

where $\mathcal{C}^{\prime}$ denotes a path around $\mathcal{C}$ lying outside the singularity at the leading edge. For the velocity field (63), the residue from the sole enclosed pole at the origin gives $X=0, Y=2 \pi \hat{\rho} U, M=\pi \hat{\rho} U$.

It is useful to derive the solution (63) and the transformation (59) from the complex potential,

$$
w_{1}(\zeta, \sigma, \tau)=(\kappa / 2 \pi \mathrm{i}) \log \left(\zeta-\sigma \mathrm{e}^{\mathrm{i} \tau}\right)-(\kappa / 2 \pi \mathrm{i}) \log \left(\zeta-\sigma^{-1} \mathrm{e}^{\mathrm{i} \tau}\right),
$$

for a point vortex of circulation $\kappa$ at $\zeta=\sigma \mathrm{e}^{\mathrm{i} \tau}$, lying inside the circle $\mathcal{C}$ when $|\sigma|<1$. For positive $\kappa$, differentiating with respect to $\sigma$ gives a dipole in the $\arg \zeta=\tau-\pi / 2$ direction [49, for example],

$$
w_{2}(\zeta, \sigma, \tau)=-(\kappa / 2 \pi \mathrm{i}) \mathrm{e}^{\mathrm{i} \tau}\left[1 /\left(\zeta-\sigma \mathrm{e}^{\mathrm{i} \tau}\right)+\sigma^{-2} /\left(\zeta-\sigma^{-1} \mathrm{e}^{\mathrm{i} \tau}\right)\right] .
$$

The dipole at the leading edge is obtained by setting $\tau=0$ and $\sigma=1$ to give

$$
w_{2}(\zeta, 1,0)=\mathrm{i} \kappa / \pi(\zeta-1),
$$

which, for $\kappa=2 \pi$, is the required complex velocity (63). 
Setting $\tau=\pi / 2$ and $\sigma=0$ gives a dipole at the origin, oriented in the $\arg \zeta=0$ direction, which, omitting an additive constant, can be written

$$
\sigma^{2} w_{2}(\zeta, 0, \pi / 2)=-(\kappa / 2 \pi)(\zeta+1 / \zeta)
$$

This is the complex potential for uniform flow past the circle but equally provides, for $\kappa=\pi$, the mapping (59) since the streamlines in the $\zeta$-plane map to lines of constant $y$ in the $z$-plane cut along the plate: the same dipole solution (70) provides the required complex velocity field and the conformal mapping. A similar result was noted by [51] when extending the results of [52] to domains of connectivity three and more, although there the correspondence was between the complex velocity potentials not between the complex velocities as here.

\subsection{A finite collection of plates}

To generalise the results of section 5.1, consider the unbounded region $D_{z}$ exterior to a collection of $M+1$ plates, denoted $D_{j}, j=0, \ldots, M$, of bounded extent aligned in the positive $x$-direction, corresponding to a set of plates at small angle of attack to an oncoming uniform flow. The domain can be scaled so that the plate $D_{0}$ lies on $y=0$ with $|x|<1$. The normal velocities on the plates are determined by their individual angles of attack and so are specified as $v_{j}$, $j=0, \ldots, M$, where $v_{0}$ can be scaled to unity by linearity. We follow [50] taking the pre-image domain to be the unit disc in the $\zeta$-plane with $M$ smaller circular discs excised, and denoting the domain by $D_{\zeta}$, the circular boundary of the $j$ th excised circular disc by $C_{j}, j=1, \ldots, M$ and the unit circle, the pre-image of $D_{0}$, by $C_{0}$. The domain is then specified by the centres $\delta_{j}$ and radii $\rho_{j}$ of the circles $C_{j}$ and the results in section 5.1 correspond to the case $M=0$. Finding the flow-field and the forces and moments on the plates thus reduces to related sub-problems: finding the conformal map between $D_{z}$ and $D_{\zeta}$ and obtaining the velocity field in $D_{\zeta}$.

The mapping between $\boldsymbol{D}_{\boldsymbol{z}}$ and $\boldsymbol{D}_{\zeta}$. The pre-image $D_{\zeta}$ is not unique but can be made so, to within a rotation, by specifying the pre-image $\zeta=\beta$ of the point $z=\infty$, at infinity in $D_{z}$. The mapping here will thus depend on $\beta$ which can be chosen to exploit, for example, symmetry in the domain $D_{z}$. The mapping from $D_{\zeta}$ to $D_{z}$ follows from the construction that led to (72). Analogously to (69), the complex velocity potential for a point vortex of strength $\kappa$ located at $\zeta_{0}=\sigma \mathrm{e}^{\mathrm{i} \tau}$ can be written [51] as

$$
G_{0}(\zeta, \sigma, \tau)=(-\mathrm{i} \kappa / 2 \pi) \log \left[\omega\left(\zeta, \sigma \mathrm{e}^{\mathrm{i} \tau}\right) / \sigma \omega\left(\zeta, \sigma^{-1} \mathrm{e}^{\mathrm{i} \tau}\right)\right]
$$

where $\omega\left(\zeta, \zeta_{0}\right)$ is the Schottky-Klein prime function associated with the domain. Differentiating (73) with respect to $\sigma$ gives a dipole with flow in the $\arg \zeta=$ $\tau-\pi / 2$ direction,

$$
w_{3}(\zeta, \sigma, \tau)=(-\mathrm{i} \kappa / 2 \pi) \mathrm{e}^{\mathrm{i} \tau}\left[\Omega\left(\zeta, \sigma \mathrm{e}^{\mathrm{i} \tau}\right)+\left(1 / \sigma^{2}\right) \Omega\left(\zeta, \sigma^{-1} \mathrm{e}^{\mathrm{i} \tau}\right)-1 / \sigma\right]
$$




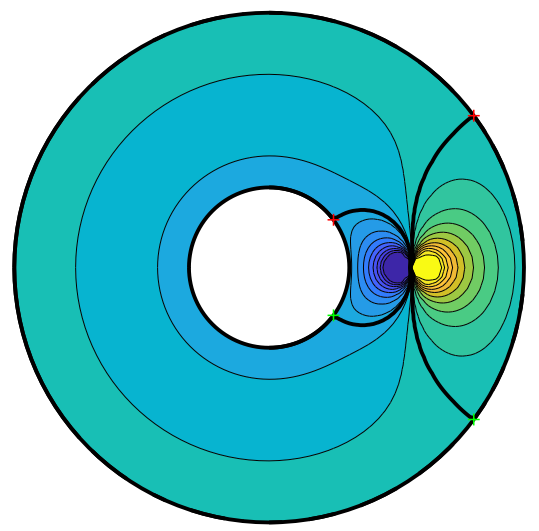

(a)

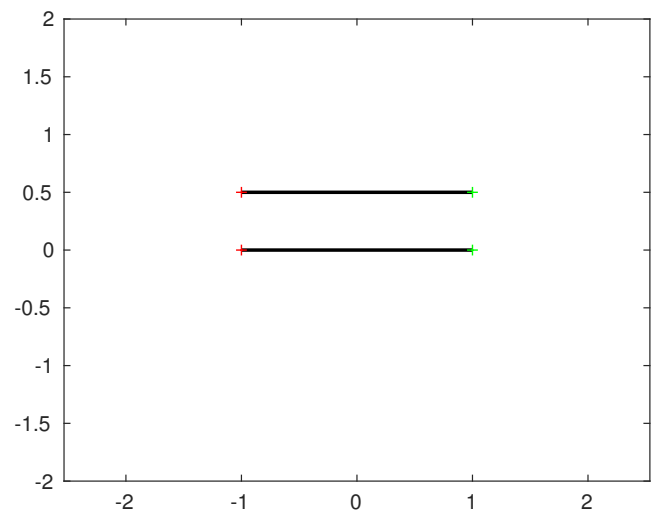

(b)

Fig. 19. The mapped domains. (a) The annular domain $D_{\zeta}$. The circular boundaries $C_{0}, C_{1}$ correspond to the plates. The dipole lies on $\Re \zeta=0$ oriented in the $\Im \zeta$ direction. Streamlines passing through the stagnation points are shown in bold with the leading edges marked by red crosses and trailing edges by green crosses. (b) The plates in the domain $D_{z}$. Streamlines here are simply lines of constant $y$.

where

$$
\Omega\left(\zeta, \zeta_{0}\right)=\frac{1}{\omega\left(\zeta, \zeta_{0}\right)} \frac{\partial \omega\left(\zeta, \zeta_{0}\right)}{\partial \zeta_{0}}
$$

is the logarithmic derivative of $\omega\left(\zeta, \zeta_{0}\right)$. Setting $\zeta_{0}=\beta$ gives a dipole at $z=\infty$, and thus uniform flow, in $D_{z}$.

It remains to find the positions $\zeta_{j l, t}=\delta_{j}+\rho_{j} \exp \left(\mathrm{i} \lambda_{j l, t}\right)$ on the circles $C_{j}$ that correspond to the leading and trailing edges of the plates. These are the pairs of points where streamlines from infinity meet the circle $C_{j}$, i.e. the stagnation points on $C_{j}$ and so satisfy

$$
\frac{\mathrm{d} \Omega}{\mathrm{d} \zeta}\left(\zeta_{j l, t}, \beta\right)=0
$$

The mapping from $D_{\zeta}$ to $D_{z}$ is straightforward given the data $\delta_{j}$ and $\rho_{j}$, with the constant value of the streamfunction $\Im w_{3}$ on $C_{j}$ giving $y_{j}$ and the values of $\Re w_{3}$ at the stagnation points giving the ends of the plates. Finding the inverse mapping is more difficult. Given the plate data it is necesssary to solve simultaneously the $M$ equations

$$
\Im w_{3}=y_{j}, \quad \text { on } \quad C_{j},
$$

and the $2 M$ equations (76). This is considered in greater detail in section 5.3.

The velocity field in $\boldsymbol{D}_{\boldsymbol{\zeta}}$. The required velocity field associated with each plate is given by a dipole tangent to the plate, i.e. tangential to the circle $C_{j}$. 


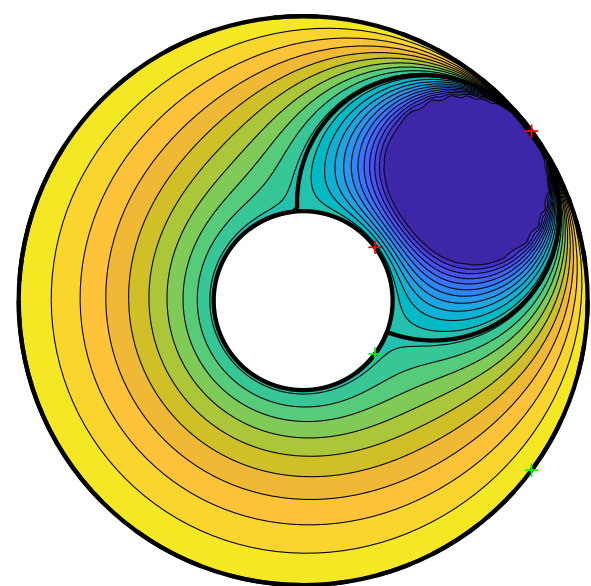

(a)

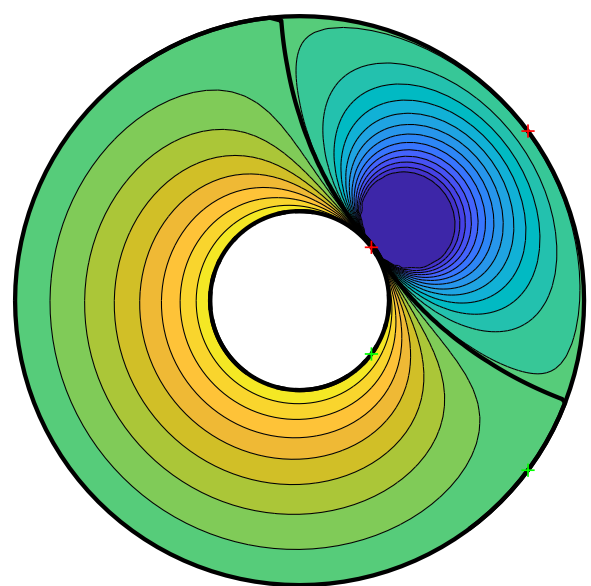

(b)

Fig. 20. Isolines in the annular domain $D_{\zeta}$ of $v$, the velocity component in the $y$ direction in the domain $D_{z}$. (a) $v=1$ on plate $D_{0}$ and $v=0$ on plate $D_{1}$. These isolines are also the streamlines for a dipole at the leading edge of plate $D_{0}$ oriented along the plate. (b) $v=0$ on plate $D_{0}$ and $v=1$ on plate $D_{1}$. These isolines are also the streamlines for a dipole at the leading edge of plate $D_{1}$ oriented along the plate. The general solution for arbitrary constant $v$ on each plate is a linear combination of these fields.

It is sufficient to construct, for each $j$, the solution that has a dipole at $\zeta_{j l}$ aligned in the $\lambda_{j l}-\pi / 2$ direction (i.e. normal to the radius at the stagnation point) with unit imaginary part on $C_{j}$ and zero imaginary part on all other boundaries. Then the solution for arbitrary constant $v$ on each plate follows by linear superposition. From (74), this is simply $w_{3}\left(\zeta,\left|\zeta_{j l}\right|, \lambda_{j l}\right)$.

\subsection{The case of two plates}

The simplest non-trivial example is given by two plates. The domain $D_{\zeta}$ can then be taken as the annulus $q<|\zeta|<1$ for some value of the conformal modulus $q$ [53]. [50] notes that for this geometry

$$
\omega(\zeta, \gamma)=-\left(\gamma / C^{2}\right) P(\zeta / \gamma, q),
$$

where

$$
P(\zeta / \gamma, q)=(1-\zeta) \prod_{k=1}^{\infty}\left(1-q^{2 k} \zeta\right)\left(1-q^{2 k} \zeta^{-1}\right), \quad C=\prod_{k=1}^{\infty}\left(1-q^{2 k}\right),
$$

so the logarithmic derivative (75), and its derivative in (76), are simple sums, allowing the solutions to (76) and (77) to be obtained straightforwardly.

Figure 19 shows the annular domain $D_{\zeta}$. The dipole for the mapping lies on $\Re \zeta=0$ oriented in the $\Im \zeta$ direction. Streamlines passing through the stagnation 
points are shown in bold with the leading edges marked by red crosses and trailing edges by green crosses. (b) The plates in the domain $D_{z}$. Streamlines here are simply lines of constant $y$. Figure 20 gives isolines in the annular domain of $v$, the velocity component in the $y$ direction in domain $D_{z}$. In (a) $v=1$ on plate $D_{0}$ and $v=0$ on plate $D_{1}$. These isolines are also the streamlines for a dipole at the leading edge of plate $D_{0}$ oriented along the plate. In (b) $v=0$ on plate $D_{0}$ and $v=1$ on plate $D_{1}$. These isolines are also the streamlines for a dipole at the leading edge of plate $D_{1}$ oriented along the plate. The general solution for arbitrary constant $v$ on each plate is a linear combination of these fields.

With the velocity field $q$ determined the forces on the plates follow from (68). Since the integrand is periodic over the closed path of integration, the trapezium rule gives exponentially accurate results. To minimise truncation errors the path is taken to lie midway between the given circle and the point, $\beta$, at infinity. Once the forces have been determined in $D_{\zeta}$ the motion of the plates in the physical plane follows the dynamics in [6]. This gives a set of ordinary differential equations that can be integrated forward in time by standard methods.

\section{$6 \quad$ Further comments}

The majority of analytical studies on dynamic fluid-body interactions have been for cases of a single body as in sections 3,4 of this chapter on a skimming body and on body liftoff respectively and in the references [1]-[11]. The exceptions are in [1] which concerns a cascade of bodies in channel flow and in the present chapter's section 5 which describes interactions involving many bodies moving in surrounding potential flow of fluid with none or possibly (by use of simple images) at most one solid fixed wall present. The aim throughout this chapter has been to seek out relatively simple configurations and basic evolution properties first, as the occurrence of important linear and nonlinear effects implies that mathematical accounts are often likely to be insightful. Assumptions made include those of unsteady laminar incompressible fluid motions over mediumto-large ranges of the Reynolds and in two spatial dimensions but there must also be awareness concerning three-dimensionality, flow separation, transition, turbulence, impacts and rebounds, and so on. We believe the study here may be of interest in terms of mathematical issues, real applications, the science of fluid dynamics with freely moving bodies present and the clear interaction with direct numerical simulations. In reality there are often very complex additional phenomena to deal with in the practical situations of concern as hinted above.

Specific issues are connected with the individual sections of the chapter. Thus considerably more detail and analysis of the original multi-body problems addressed in section 2.1 is to be found in [10] especially in terms of the wakes behind the bodies but also in terms of the influences from the presence of flexible patches of wall which respond to the local pressure due to the fluid flow: compare with [7]. More investigation of the skimming problems described in section 2.2 and later in section 3 is given in [9]. It would be interesting to consider adding 
in the effects of three-dimensionality within the various skimming-body analyses conducted to date and the same comment applies to the analysis of liftoff. Both for the skimming scenario in section 3 and the liftoff scenario in section 4 the influence of viscosity also remains to be examined seriously given that the influence is potentially substantial. As far as the beginnings of many-body analysis in section 5 are concerned the basic elements of an account that includes viscous effects can be found in [6] for the channel-flow situation, where essentially the same viscous-inviscid interplay over two axial length scales covers the case of many short bodies at small incidence, while the corresponding situation for external flow with a boundary layer brings in triple-deck theory. An extension of the study in reference [7] would be necessary to cater for many long bodies freely moving inside a boundary layer or channel flow.

There are numerous intriguing follow-on studies which could be undertaken. With that in mind we refer to the earlier remarks on three-dimensional effects, on many bodies being present in the fluid flow and on the influences of different body lengths as well as thicknesses. In addition future investigations could tackle increased connections with experimental work, observations and direct numerical simulations, not to forget full nonlinearity arising with a body in a viscous wall layer or with a body of length comparable with the development length in the oncoming flow, namely of the order of the chord length in a boundary layer and of order Reynolds number multiplied by the channel width in a channel flow. Body movement relative to the nearby wall can also be a key factor [7]. We should perhaps mention that there are many other near-wall phenomena of interest, including problems of flexible walls, dynamic roughnesses on a surface and the impact on airfoil stall. Finally, novel instabilities occur in most of the dynamic fluid-body interactions addressed so far, and these instabilities merit much further study.

Acknowledgements. Thanks for support are due (FTS) to EPSRC through grant numbers GR/T11364/01, EP/G501831/1, EP/H501665/1 during part of this research, and (SB) to the Republic of Turkey for financial support. The authors would like to thank the Isaac Newton Institute for Mathematical Sciences for support and hospitality during the programme The Mathematics of Sea Ice Phenomena when part of the work on this paper was finalised, supported by EPSRC grant number EP/K032208/1. Thanks are due to Roger Gent and Richard Moser at AeroTex, Rob Lewis at TotalSim, Sarah Bee and Mark Honeywood at Sortex-Buhler and UCL colleagues Robert Bowles, Nick Ovenden and Sergei Timoshin for very helpful discussions on body and particle movement in near-wall shear flow.

\section{References}

1. Smith, F. T., Ellis, A. S.: On interaction between falling bodies and the surrounding fluid, Mathematika, 56:140-168, (2010)

2. Hicks, P. D., Smith, F. T.: Skimming impacts and rebounds on shallow liquid layers, Proc. R. Soc. A, 467, 653-674, (2011) 
3. Smith, F. T., Wilson, P. L.: Fluid-body interactions: clashing, skimming, bouncing, Phil. Trans. R. Soc. A, 369, 3007-3024, (2011)

4. Smith, F. T., Wilson, P. L.: Body-rock or lift-off in flow. J. Fluid Mech., 735:91-119, (2013)

5. Liu, K., Smith, F. T.: Collisions, rebounds and skimming. Phil. Trans. R. Soc. A, $372(2020),(2014)$

6. Smith, F. T., Johnson, E. R.: Movement of a finite body in channel flow, Proc. R. Soc. A 472(2191): 20160164, (2016)

7. Smith, F. T.: Free motion of a body in a boundary layer or channel flow, J. Fluid Mech., 813:279-300, (2017)

8. Smith, F. T., Liu, K.: Flooding and sinking of an originally skimming body, J. Eng. Math. 107(1), 37-60, (2017)

9. Liu, J.: Shallow-water skimming, skipping and rebound problems, Ph.D. thesis, University College London. (2017)

10. Balta, S.: On fluid-body and fluid-network interactions, Ph.D. thesis, University College London. (2017)

11. Balta, S., Smith, F. T.: Fluid flow lifting a body from a solid surface. In preparation. (2018)

12. Ladd, A. J. C. : Numerical simulations of particulate suspensions via a discretized Boltzmann equation. Part 2. Numerical results. J. Fluid Mech., 271, 311-339, (1994)

13. Foucaut, J. -M., Stanislas, M.: Take-off threshold velocity of solid particles lying under a turbulent boundary layer. Experiments in Fluids, 20(5), 377-382, (1996)

14. Patankar, N. A., Huang, P. Y., Ko, T., Joseph, D. D.: Lift-off of a single particle in Newtonian and viscoelastic fluids by direct numerical simulation. J. Fluid Mech., 438, 67-100, (2001)

15. Gray, J. M. N. T., Ancey C.: Multi-component particle-size segregation in shallow granular avalanches, Journal of Fluid Mechanics, 678, 535-588, (2011)

16. Virmavirta, M., Kivekäs J., Komi P. V.: Take-off aerodynamics in ski jumping, Journal of Biomechanics, 34(4), 465-470, (2001)

17. Miller, M. C., McCave, I. N., Komar, P. D.: Threshold of sediment motion under unidirectional currents, Sedimentology, 24(4), 507-527, (1977)

18. Owen, P. R.: Saltation of uniform grains in air, Journal of Fluid Mechanics, 20(2), 225-242, (1964)

19. Shao, Y., Raupach, M. R., Findlater, P. A.: Effect of saltation bombardment on the entrainment of dust by wind, Journal of Geophysical Research: Atmospheres, 98(D7), 12719-12726, (1993)

20. Jia, L.-B., Li, F., Yin, X.-Z., Yin, X.-Y.: Coupling modes between two flapping filaments. J. Appl. Mech., 581, 199-220, (2007)

21. Guazzelli, E.: Sedimentation of small particles: how can such a simple problem be so difficult? C.R. Mećanique, 334, (2006)

22. Godone, D., Stanchi, S.: Soil Erosion Studies, InTech, (2011)

23. Andrew, M., David, M., deVeber G., Brooker, L. A.: Arterial thromboembolic complications in paediatric patients. Thromb. Haemost. 78(1), 715-725, (1997)

24. Babyn, P. S., Gahunia, H. K., Massicotte, P.: Pulmonary thromboembolism in children. Pediatr. Radiol. 35(3), 258-274, (2005)

25. Baker, W. F. Jr.: Diagnosis of deep venous thrombosis and pulmonary embolism. Med. Clin. North Am. 82(3), 459-476, (1998)

26. Gaver III, D. P., Jensen O. E., Halpern, D.: Surfactant and airway liquid flows. In Lung Surfactant and Disorder: Lung Biology in Health and Disease (ed. K. Nag), Taylor and Francis, 201,191, (2005) 
27. Iguchi, Y., Kimura, K.: A case of brain embolism during catheter embolisation of head arteriovenous malformation. What is the mechanism of stroke? J. Neurol. Neurosurg. Psychiatry 78(1), 81, (2007),

28. Secomb, T. W., Skalak, R., Özkaya, N., Gross, J. F.: Flow of axisymmetric red blood cells in narrow capillaries. J. Fluid Mech. 163, 405-423, (1986)

29. White, A. H.: Mathematical modelling of the embolisation process in the treatment of arteriovenous malformations. PhD Thesis, University of London. (2008)

30. Balta, S., Smith, F. T.: Inviscid and low viscosity flows in multi-branching and reconnecting networks. J. Eng. Math. 104(1), 1-18, (2017)

31. Ellis, A. S.: Modelling chute delivery of grains in a food-sorting process, Ph.D. Thesis, University of London, (2007)

32. Ellis, A. S., Smith, F. T.: A continuum model for a chute flow of grains. SIAM J. Appl. Math., 69(2), 305-329, (2008)

33. Koch, D. L., Hill, R. J.: Inertial effects in suspension and porous-media flows. Annu. Rev. Fluid Mech., 33, 619, (2001)

34. Willetts, B.: Aeolian and fluvial grain transport. Philos.Trans. R. Soc. Lond. A, 356(1747), 2497-2513, (1998)

35. Bowles R. G. A., Smith, F. T.: Lifting multi-blade flows with interaction. J. Fluid Mech. 415, 203-226, (2000)

36. Purvis R., Smith, F. T.: Planar flow past two or more blades in ground effect. Q. J. Mech. Appl. Math. 57(1), 137-160, (2004)

37. Smith, F. T., Timoshin, S. N.: Planar flows past thin multi-blade configurations. J. Fluid Mech. 324, 355-377, (1996b)

38. Smith, F. T., Ovenden, N. C., Franke, P. T., Doorly, D. J.: What happens to pressure when a flow enters a side branch? J. Fluid Mech. 479, 231-258, (2003)

39. Tuck, E. O., Dixon, A.: Surf-skimmer planing hydrodynamics, J. Fluid Mech. 205, 581-592, (1989)

40. Smith, F. T.: Upstream interactions in channel flows, J. Fluid Mech. 79, 631-655, (1977)

41. Howison, S. D., Ockendon, J. R., Oliver, J. M.: Oblique slamming, planing and skimming, J. Eng. Math., 48, 321-337, (2004)

42. Khabakhpasheva, T. I., Korobkin, A. A.: Oblique impact of a smooth body on a thin layer of liquid, Proc. R. Soc., 469(2151), (2013)

43. Wagner, H.: Über Stoß-und gleitvorgänge an der Oberflaäche von Flüssigkeiten (Phenomena associated with impacts and sliding on liquid surfaces), Zeitschr. Math. Mech., 12, (1932)

44. Batyaev, E. A., Khabakhpasheva, T. I.: Initial stage of the inclined impact of a smooth body on a thin fluid layer, Fluid Dynamics, 48(2), 211-222, (2013)

45. Stewartson, K., Williams, P. G.: Self-induced separation, Proc. R. Soc. Lond., A 312(1509), 181-206, (1969)

46. Smith, F. T.: The Laminar Separation of an Incompressible Fluid Streaming Past a Smooth Surface, Proc. R. Soc. Lond., 356(1687), 443-463, (1977)

47. Scheichl, B., Kluwick, A., Smith, F. T.: Break-away separation for high turbulence intensity and large Reynolds number, J. Fluid Mech., 670, 260-300, (2011)

48. Lagally, M.: Die reibungslose Strömung im Aussengebiet zweier Kreise, Z. Angew. Math. Mech., 9, 299-305, (1929)

49. Johnson, E. R. and McDonald, N. R.: The motion of a vortex near two circular cylinders, Proc. Roy. Soc. A, 460, 939-954, (2004)

50. Crowdy, D. G.: Calculating the lift on a finite stack of cylindrical aerofoils, Proc. Roy. Soc. A, 462, 1387-1407, (2006) 
51. Crowdy, D. G. and Marshall, J. S.: Analytical formulae for the Kirchhoff-Routh path function in multiply connected domains, Proc. Roy. Soc. A, 461, 2477-2501, (2005)

52. Johnson, E. R. and McDonald, N. R.: Vortices near barriers with multiple gaps, J. Fluid Mech., 531, 335-358, (2005)

53. Nehari, Z.: Conformal mapping, McGraw-Hill, (1952) 